\title{
Research Square \\ VTR: an algorithm for identifying analogous contacts on protein structures and their complexes
}

\section{Vitor Pimentel}

Universidade Federal de Minas Gerais

Diego Mariano ( $\nabla$ diegomariano@ufmg.br)

Universidade Federal de Minas Gerais https://orcid.org/0000-0002-5899-2052

\section{Letícia Xavier Silva Cantão}

Universidade Federal de Minas Gerais

\section{Luana Luiza Bastos}

Universidade Federal de Minas Gerais

\section{Pedro Fischer}

Universidade Federal de São João Del-Rei

Leonardo Henrique França de Lima

Universidade Federal de São João Del-Rei

\section{Alexandre Victor Fassio}

Universidade Federal de Minas Gerais

Raquel Cardoso de Melo-Minardi ( $\sim$ raquelcm@dcc.ufmg.br)

Universidade Federal de Minas Gerais https://orcid.org/0000-0001-5190-100X

\section{Research Article}

Keywords: protein interactions, structural bioinformatics, structural alignment, contacts, rational design of enzymes

Posted Date: December 1st, 2020

DOI: https://doi.org/10.21203/rs.3.rs-82599/v2

License: (c) (i) This work is licensed under a Creative Commons Attribution 4.0 International License.

Read Full License 
1 VTR: an algorithm for identifying analogous contacts on protein

\section{structures and their complexes}

3 Vitor Pimente ${ }^{1 \dagger}$, Diego Mariano ${ }^{1 \dagger *}$, Letícia Xavier Silva Cantão ${ }^{1}$, Luana Luiza Bastos ${ }^{1}$, Pedro

4 Fischer $^{2}$, Leonardo Henrique França de Lima $^{2}$, Alexandre Victor Fassio ${ }^{1}$, Raquel Cardoso de

5 Melo-Minardi ${ }^{*}$

$6 \quad{ }^{1}$ Laboratory of Bioinformatics and Systems. Department of Computer Science. Universidade Federal de Minas

7 Gerais, Belo Horizonte, 31270-901, Brazil.

$8{ }^{2}$ Laboratory of Molecular Modelling and Bioinformatics (LAMMB), Department of Physical and Biological

9 Sciences, Universidade Federal de São João Del-Rei, Campus Sete Lagoas, Sete Lagoas, 35701-970, Brazil.

$10 \dagger$ These authors contributed equally to this work.

11 *To whom correspondence should be addressed: RCMM: raquelcm@dcc.ufmg.br; DM: diegomariano@ufmg.br 


\section{Abstract}

13 Evolutionarily related proteins can present similar structures but very dissimilar sequences.

14 Hence, understanding the role of the inter-residues contacts for the protein structure has been

15 the target of many studies. Contacts comprise non-covalent interactions, which are essential to

16 stabilize macromolecular structures such as proteins. Here we show VTR, a new method for

17 the detection of analogous contacts in protein pairs. VTR performs structural alignment

18 between proteins and detects interactions that occur in similar regions. To evaluate our tool,

19 we proposed three case studies: (i) we compared a vertebrate myoglobin and a truncated

20 invertebrate hemoglobin; (ii) analyzed interactions between the spike protein RBD of SARS-

$21 \mathrm{CoV}-2$ and the cell receptor ACE2; and (iii) compared a glucose-tolerant and a non-tolerant $\beta$ -

22 glucosidase enzyme used for biofuel production. The case studies demonstrate the potential of

23 VTR for the understanding of functional similarities between distantly sequence-related

24 proteins, as well as the exploration of important drug targets and rational design of enzymes

25 for industrial applications. We envision VTR as a promising tool for understanding differences

26 and similarities between homologous proteins with similar 3D structures but different

27 sequences. VTR is available at http://bioinfo.dcc.ufmg.br/vtr.

29 Keywords: protein interactions; structural bioinformatics; structural alignment; contacts;

30 rational design of enzymes 


\section{Introduction}

32 Proteins are macromolecules responsible for most functions in living beings, such as transport,

33 immune protection, control growth, and so on ${ }^{1}$. The function of a protein is directly related to

34 their three-dimensional structure. Previous studies have demonstrated that structural changes

35 are directly related to sequence changes ${ }^{2}$. Even small modifications, such as mutations,

36 insertions, or deletions, can change the structure ${ }^{3-5}$. However, evolutionarily related proteins

37 may present similar structures but different sequences ${ }^{6}$. Sequence alignments can unambiguously distinguish similar and non-similar structures when the identity is upper to $40 \%$. Even sequences with identities between $20-35 \%$ may generate false negatives for homology identification ${ }^{7}$. Also, studies have reported similarities in structures with sequence

41 identity lower than $20 \%{ }^{2}$. Until now, the understanding of how the polypeptide sequences fold 42 into a particular three-dimensional shape after synthesis remains a mystery ${ }^{8,9}$. It has motivated 43 the search for computational algorithms to predict protein structures from their sequences ${ }^{10,11}$ 44 or even detect and annotate correctly protein functions ${ }^{12-14}$. Besides, understanding protein structure and their interactions accurately are crucial to the rational design of molecules for several applications, including discovering novel drugs and improving enzymes for the 47 biotechnological industry ${ }^{15,16}$.

Thus, understanding the role of inter-residues contacts in protein folding, stabilization, and function has been the goal of several studies ${ }^{17,18}$. Contacts are weak and potentially stabilizing interactions in the structure of macromolecules, such as proteins ${ }^{18,19}$. They can be hydrophobic interactions, electrostatic attractive or repulsive interactions, disulfide bonds, aromatic stacking, hydrogen bonds, and so on ${ }^{17,20-22}$. Contacts also have been used to compare

53 protein structures, for instance, from contact maps ${ }^{1}$ or graph-based structural signatures ${ }^{23}$.

54 Recent computational approaches have suggested that the substitution of non-interacting residues for interacting ones can improve protein stability, which highlights the importance of 
computation of contacts ${ }^{16}$. In addition, pairwise comparisons between proteins can be performed using visual and structural alignment tools, such as PyMOL ${ }^{24}$. However, comparisons between contacts are usually performed individually, which makes comparisons between a considerable number of contacts toilsome. To the best of our knowledge, there is no tool for systematic structural comparisons between contacts in a protein pair.

Therefore, herein, we propose a novel approach to detect and align contacts for protein structure analysis and pairwise comparison. Our algorithm aims to detect differences and similarities in amino acid residues pairs in contact when compared to analogous positions. For this purpose, we first perform a structural superposition between two proteins, detect contacts through a cutoff-based approach, and compare contacts in analogous positions using a score. We also developed a user-friendly web tool called VTR to facilitate the use of our method. As a proof of concept, we propose three case studies: (i) a comparison between a myoglobin (PDB ID: 1a6m) and a hemoglobin (PDB ID: 1dlw), proteins with similar structure but low sequence identity (18\%); (ii) a comparison of interactions among the spike protein RBD of SARS-CoV2, SARS-CoV, and the cell receptor ACE2; and (iii) a comparison between a glucose-tolerant $\beta$-glucosidase enzyme efficient for biofuel production (Bgl1A) and a less efficient non-tolerant $\beta$-glucosidase (Bgl1B).

\section{Results and discussion}

\section{VTR tool workflow}

6 VTR receives as input two PDB files (hereafter called A and B). We suggest that PDBs present similar folding, but if structures with different folds were used, VTR would try to perform the best structural alignment using the TM-align tool. It rotates and translates the coordinates of the protein A considering the alignment with B. Then, VTR uses in-house Python scripts to calculate contacts: (i) hydrogen bonds; (ii) salt bridge (hydrogen bonds and attractive); (iii) 
81 ionic interaction (attractive); (iv) repulsive; (v) hydrophobic interactions; (vi) aromatic

82 stacking; and (vii) disulfide bonds. Then, VTR determines contacts in analogous positions

83 using AVD score (average distance between the coordinates of atoms in contact), performs

84 comparative statistical, and returns the results for the VTR interface (Figure 1 A-C).

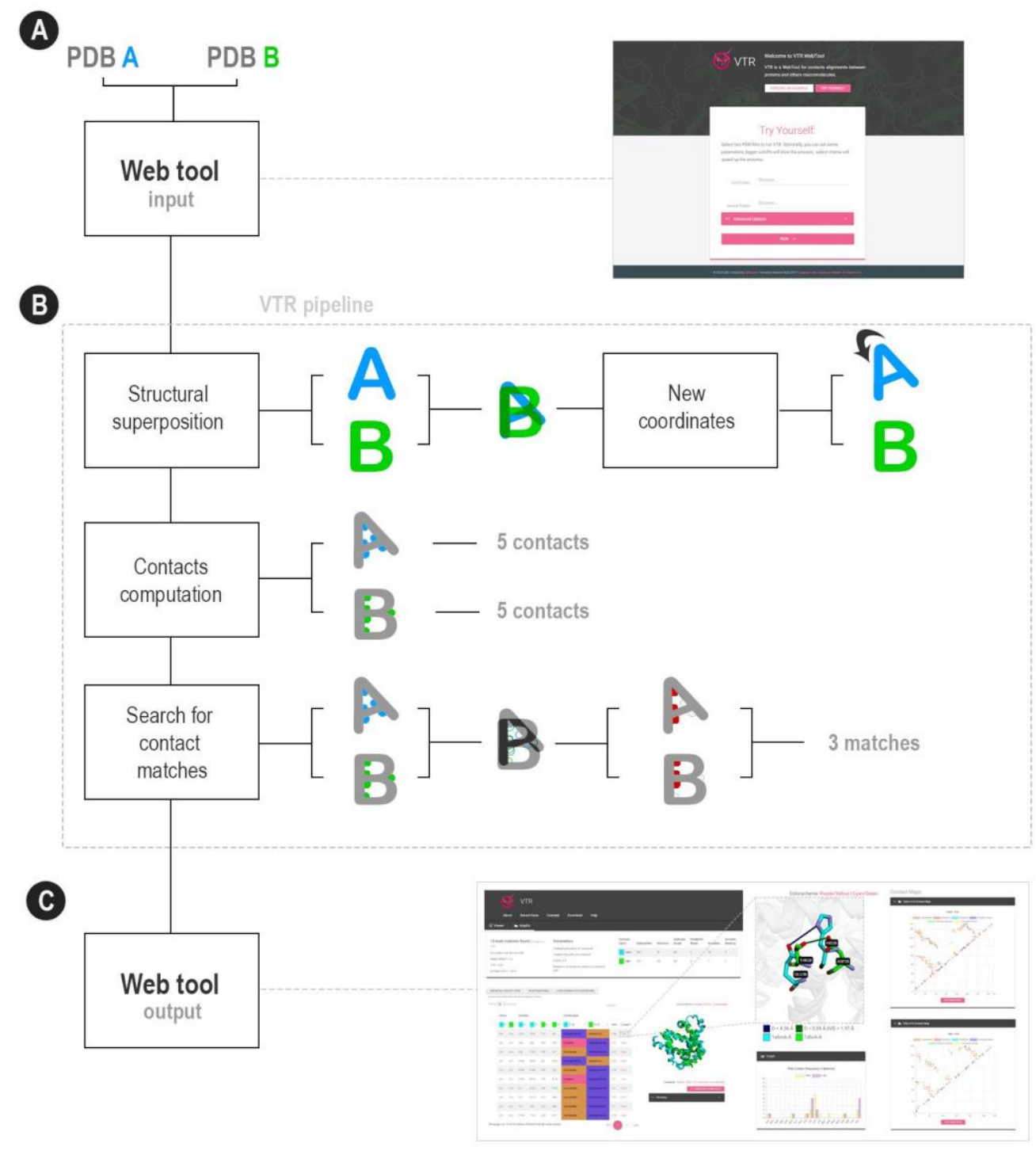

86 Figure 1. VTR workflow. (a) VTR web tool receives as input two PDB files. (b) VTR pipeline analyzes the PDB files in three steps: (i) structural superposition between the PDB files using TM-align; (ii) contacts calculation using cutoff definitions obtained from the literature; and (iii) search for analogous contacts using AVD strategy. (c) VTR returns the contact matches, and users can use them in the web interface. Also, VTR determines the statistics of differences between amino acids in contact. 


\section{Case studies}

94 To evaluate the VTR tool, we proposed three pairwise comparative case studies: (i) eukaryotic

95

96

97

98

99

100

101

102

103 myoglobin and a truncated non-vertebrate hemoglobin chain; (ii) interactions among the cell receptor ACE2 and both SARS-CoV-2 and SARS-CoV; and (iii) glucose-tolerant and nontolerant $\beta$-glucosidases. Contact maps generated by VTR demonstrate similarities in the contact pattern between protein pairs in each case study. For instance, Figure 2A is more similar to Figure $2 \mathrm{~B}$, Figure $2 \mathrm{C}$ to $\mathrm{D}$, and Figure $2 \mathrm{E}$ to $\mathrm{F}$. We aim with these case studies to demonstrate a simple use for VTR (case study 1) and show the tool's effectiveness in finding shared and unshared contacts in two systems already described in the literature (case study 2). In the third case study, we propose a real use of VTR at detecting possible mutation sites for enhancing enzymes with biotechnological applications.
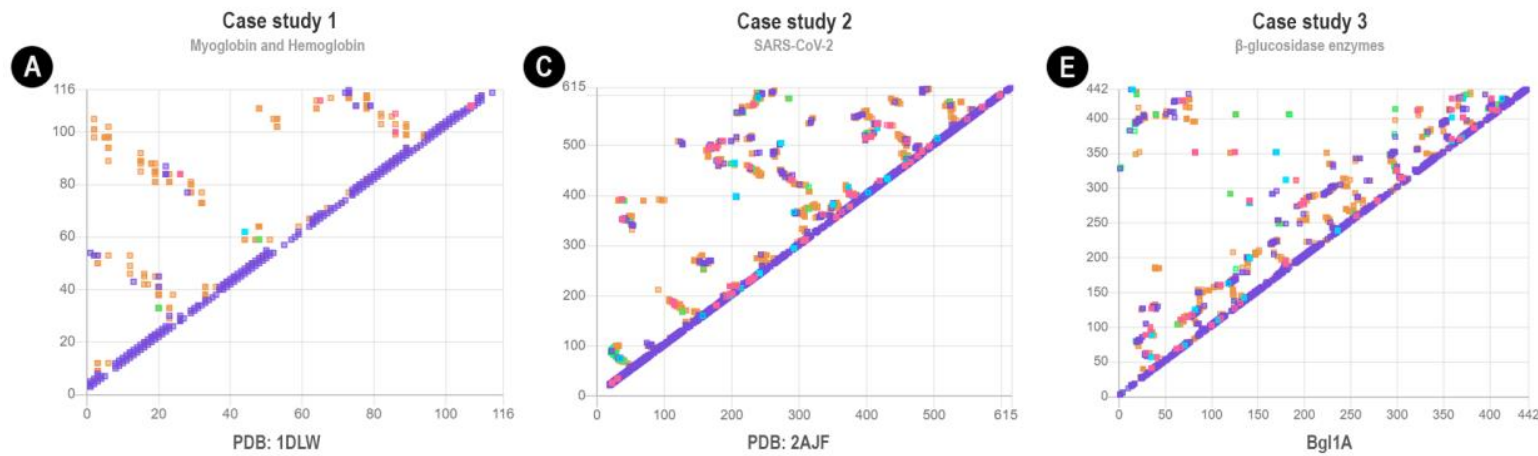

B

(D)
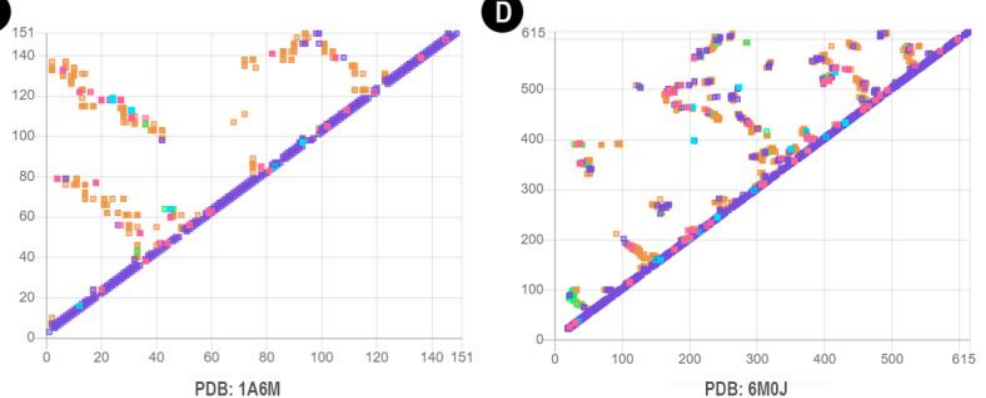

$\boldsymbol{\theta}$

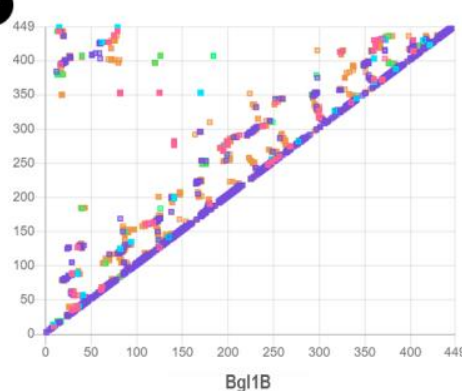

104

Figure 2. Contact maps for case studies 1 (a-b), 2 (c-d), and 3 (e-f). Each point represents a contact. Contacts of similar types are shown in the same colors. In the $x$-axes and $y$-axes are shown the residue numbers. (a) PDB ID: 1DLW; (b) PDB ID: 1A6M; (c) PDB ID: 2AJF; (d) PDB ID: 6M0J; (e) Bgl1A; (f) Bgl1B. For $C$ and $D$, we show only contact maps for chain $A$. We consider all contacts for determination of the contact maps, including contacts between atoms of the main-chain of closer residues (such as those present in alpha-helices). 


\section{Case study 1: Myoglobin and hemoglobin}

113 In the first case study, we performed the contacts alignment between a sperm whale myoglobin

114 (PDB ID: 1a6m) and a truncated single-chain hemoglobin from Paramecium caudatum ciliated

115 protozoan (PDB ID: 1dlw). Myoglobins and hemoglobins are both oxygen-binding proteins,

116 belonging to the widespread and distantly related globin family ${ }^{25}$. For this case study, the

117 evaluated myoglobin structure $(1 \mathrm{a} 6 \mathrm{~m})$ was at the oxy state (i.e., oxygen bound), while the

118 hemoglobin structure (1dlw) was at the de-oxy one (i.e., oxygen unbound). 1a6m presents a

119 sequence length of 151 amino acids, while $1 \mathrm{dlw}$, as a typical non-vertebrate truncated

120 hemoglobin chain, has a minor amino acid content, with just 116 residues. Both proteins

121 present a similar folding but a sequence identity of only $18 \%$. The literature has described that structures of homologous sequences with an identity lower than $20 \%$ may present large structural differences ${ }^{2}$. However, the discrepancy about this typical strong relationship

124 between sequence and folding similarity for the globin family has long been known. In fact,

125 globins form a family substantially conserved in structural topology, despite the distant sequence relationship, being this one of the higher conundrums in biochemistry ${ }^{3,25}$. Hence, we believe these proteins to be potential targets for comparison of analogous contacts using VTR. contacts for 1a6m: 85 hydrogen bonds, 81 attractive interactions, 32 repulsive interactions, nine

130 aromatic stacking, and 364 hydrophobic interactions. We found the following contacts in $1 \mathrm{dlw}$ :

13165 hydrogen bonds, 20 attractive interactions, one repulsive interaction, two aromatic stacking, and 221 hydrophobic interactions. However, we obtained only 13 main contact matches in analogous positions using a $2 \AA$ AVD cutoff (Table S2). The contact matches increase to 60

134 when considering conserved contact matches of hydrophobic type. 
an attractive contact between H36 and E38. However, it detected a hydrogen bond between

138 D26 and T28 in analogous positions of 1dlw (Figure 3B). On the other hand, in 1a6m, I75

139 performs hydrophobic interactions with L86, while F48 performs the same type of interaction

140 with V109 (Figure 3C). Interestingly, I75 and L86 are located at a distance of 11 amino acids,

141 while F48 and V109 are at a distance of 61 positions. This highlights the VTR's ability to detect

142 contacts even in different sequence positions. Most of the contact matches are located at

143 compatible distances of 2 to 4 amino acids, such as K102-F106 and A76-T80 (Figure 3D),

144 L104-S108 and F78-I82 (Figure 3E), L89-H93 and L64-H68 (Figure 3F). Only the hydrogen

145 bond detected in the contact L89-H93 of 1a6m was considered a match with Q13-N43. This

146 may suggest that both contacts present similar importance for the stability of both proteins.

A

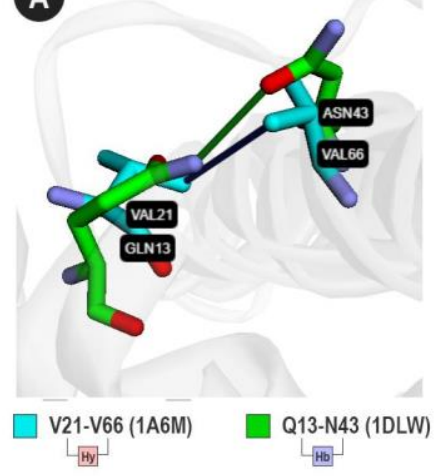

(D)

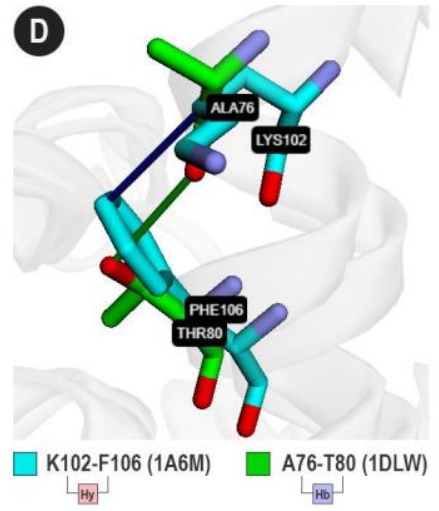

B

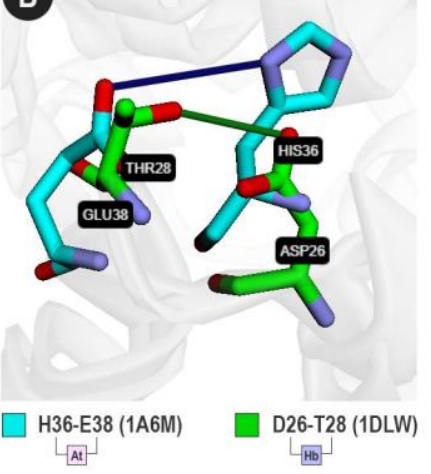

(

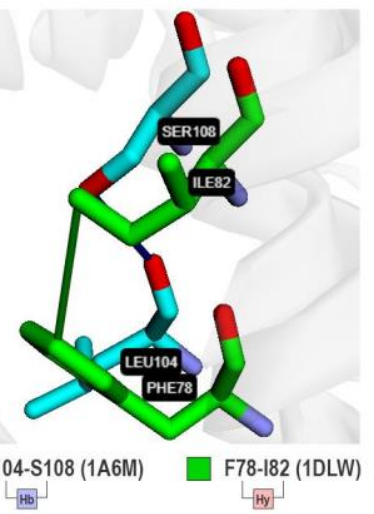

C
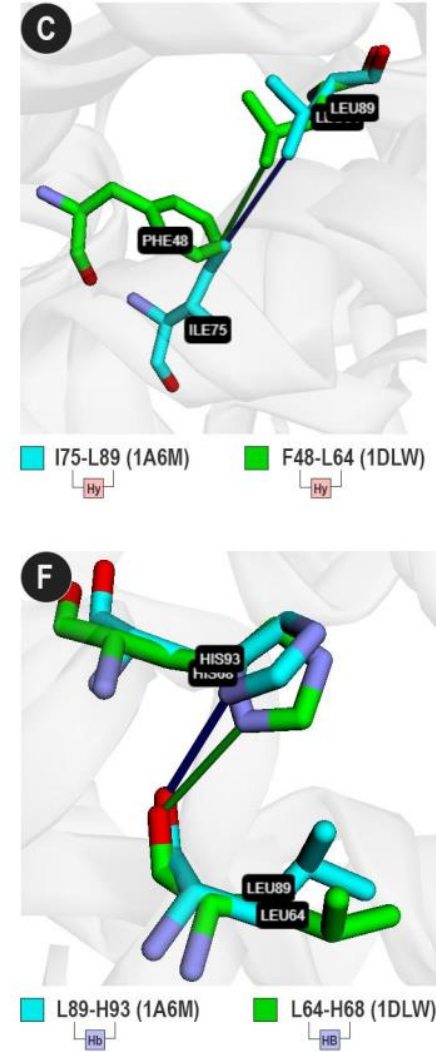

Figure 3. Six analogous contacts between $1 \mathrm{a} 6 \mathrm{~m}$ (cyan sticks) and 1dlw (green sticks). [Hy] hydrophobic; [Hb] Hydrogen bond; [At] Attractive ionic interaction. (a) V21-V66 (1a6m) and Q13-N43 (1dlw); (b) H36-E38 (1 a6m) and D26-T28 (1 dlw); (c) I75-L89 (1dlw) and F48-L64 (1dlw); (d) K102-F106 (1a1m) and A76-T80 (1dlw); (e) L104-S108 (1a6m) and F78-I82 (1dlw); and (f) L89-H93 (1a6m) and L64-H68 (1dlw). Sticks were colored using the cyan/green color scheme. 
153

154

155

156

157

158

159

160

161

162

163

164

165

166

167

168

169

170

171

172

173

174

175

176

177

It is essential to highlight that for this analysis, we did not consider the interactions between atoms of the main-chain of closer residues. We did it to reduce the number of contacts detected in alpha-helices, which can explain the low number of hydrogen bonds conserved. Enabling the advanced option "detection of structural contacts in $\alpha$-helices", the number of hydrogen bond contact matches increases to 129 . This option is disabled by default because VTR desires highlight conserved interactions with the side-chain atoms. Also, enabling this option increases the computational cost once that all contacts of similar secondary structures in close regions will be computed.

\section{Case study 2: Analyzing interactions between the spike protein RBD of SARS-CoV-2 and}

\section{the cell receptor ACE2}

Recently, a new coronavirus (SARS-CoV-2) was related to severe acute respiratory syndrome (COVID-19), spreading rapidly worldwide and causing a pandemic situation ${ }^{26}$. A sequence comparison demonstrates that SARS-CoV-2 structures share approximately $80 \%$ of sequence identity with the SARS-CoV ${ }^{26,27}$. Like SARS-CoV, SARS-CoV2 recognizes the ACE2 (Angiotensin-Converting Enzyme 2) receptor in humans. Hence, understanding the binding mechanism of the spike RBD (Receptor-Binding Domain) of SARS-CoV-2 with ACE2 may help to shed some light on the mechanism of recognition of virus receptors and the initial infection process. In a recent study, Lan et al. ${ }^{27}$ identified the relevant residues to the interaction of SARS-CoV-2 with the receptor. It was noted that most of these residues are highly conserved and shared with SARS-CoV. Here, we verified the ability of VTR to find the known contacts between different chains of both structures. We compared the structures of SARS-CoV-2 spike RBD (PDB ID 6m0j) and SARS-CoV spike RBD (PDB ID 2ajf), both in complex with the ACE2 receptor. We maintain the standard $2 \AA$ AVD cutoff, and we use chain A (ACE2) and chain E (RBD portion of the virus) for both structures. 
179 SARS-CoV and ACE2 (3 salt bridges and 13 hydrogen bonds). VTR could also find 15 contacts

180 between SARS-CoV-2 and ACE2 (2 salt bridges and 13 hydrogen bonds). Of these, VTR

181 calculated and presented which contacts were shared between receptor and SARS-CoV or

182 SARS-CoV-2. We detected all contacts described in ${ }^{27}$ (Table 1).

183 Table 1. Analogous contacts between proteins with PDB entries SARS-CoV $(6 \mathrm{~m} 0 \mathrm{j})$ and 2ajf. R1: 184 residue 1; R2: residue 2; HB: hydrogen bonds, HY: hydrophobic. In the last column, we highlighted lines 185 where the matches were reported in ${ }^{27}$. Residues in contact, but atom interactions were not described 186 in ${ }^{27}$, are presented as "-".

\begin{tabular}{|c|c|c|c|c|c|c|c|}
\hline & \multicolumn{4}{|c|}{ Chain:Residue (atom) } & \multicolumn{2}{|c|}{ Contact type } & \multirow[b]{2}{*}{$\begin{array}{l}\text { Reported } \\
\text { in } 27\end{array}$} \\
\hline & $\begin{array}{c}\text { SARS-CoV-2 } \\
\text { (R1) }\end{array}$ & $\begin{array}{l}\text { SARS-CoV-2 } \\
\text { (R2) }\end{array}$ & $\begin{array}{c}\text { SARS-CoV } \\
\text { (R1) }\end{array}$ & $\begin{array}{c}\text { SARS-CoV } \\
\text { (R2) }\end{array}$ & $\begin{array}{l}\text { SARS- } \\
\text { CoV-2 } \\
\text { R1-R2 }\end{array}$ & $\begin{array}{c}\text { SARS } \\
\text {-CoV } \\
\text { R1-R2 }\end{array}$ & \\
\hline 1 & $\mathrm{~A}: \mathbf{Y 4 1}(\mathrm{OH})$ & E:N501 (N) & $\mathrm{A}: Y 41(\mathrm{OH})$ & $\mathrm{E}: \mathbf{T 4 8 7}(\mathrm{N})$ & $\mathrm{HB}$ & $\mathrm{HB}$ & $\checkmark$ \\
\hline 2 & $\mathrm{~A}: \mathrm{Y41}(\mathrm{OH})$ & E:N501 (OD1) & A:Y41 (CE2) & E:T487 (CG2) & HB & $\mathrm{HY}$ & - \\
\hline 3 & A:D38 (OD2) & E:Y449 (OH) & A: D38 (OD2) & E:Y436 (OH) & $\mathrm{HB}$ & $\mathrm{HB}$ & $\checkmark$ \\
\hline 4 & A:Y83 $(\mathrm{OH})$ & E:N487 (OD1) & A:Y83 $(\mathrm{OH})$ & E:N473 (ND2) & HB & HB & $\checkmark$ \\
\hline 5 & A:H34 (NE2) & $\mathrm{E}: Y 453(\mathrm{OH})$ & A:H34 (NE2) & $\mathrm{E}: \mathrm{Y440}(\mathrm{OH})$ & $\mathrm{HB}$ & $\mathrm{HB}$ & \\
\hline 6 & A:E37 (OE2) & E:Y505 $(\mathrm{OH})$ & A:E37 (OE1) & E:Y491 (OH) & $\mathrm{HB}$ & $\mathrm{HB}$ & \\
\hline 7 & A:D38 (OD1) & $\mathrm{E}: \mathrm{Y} 449(\mathrm{OH})$ & A:D38 (OD1) & $\mathrm{E}: \mathrm{Y} 436(\mathrm{OH})$ & HB & HB & - \\
\hline 8 & A:G354 (O) & $\mathrm{E}: \mathbf{G 5 0 2}(\mathrm{N})$ & A:G354 (O) & $\mathrm{E}: \mathbf{G 4 8 8}(\mathrm{N})$ & HB & HB & \\
\hline 9 & $\mathrm{~A}: \mathbf{F} 28(\mathrm{~N})$ & $\mathrm{E}: Y 489(\mathrm{OH})$ & $\mathrm{A}: \mathbf{F 2 8}(\mathrm{N})$ & E:Y475 (OH) & $\mathrm{HB}$ & $\mathrm{HB}$ & \\
\hline 10 & A:Y83 $(\mathrm{OH})$ & $\mathrm{E}: Y 489(\mathrm{OH})$ & A:Y83 $(\mathrm{OH})$ & $\mathrm{E}: Y 475(\mathrm{OH})$ & $\mathrm{HB}$ & $\mathrm{HB}$ & $\checkmark$ \\
\hline 11 & A:Q42 (NE2) & $\mathrm{E}: Y 449(\mathrm{OH})$ & $\mathrm{A}: \mathbf{Q 4 2}(\mathrm{OE} 1)$ & $\mathrm{E}: Y 436(\mathrm{OH})$ & HB & HB & \\
\hline 12 & A:Y41 (OH) & E:T500 (OG1) & A:Y41 $(\mathrm{OH})$ & E:T486 (OG1) & HB & HB & $\checkmark$ \\
\hline 13 & A:Q24 (OE1) & E:N487 (ND2) & A:Q24 (OE1) & E:N473 (ND2) & HB & $\mathrm{HB}$ & $\checkmark$ \\
\hline 14 & A:K353 (O) & $\mathrm{E}: \mathbf{G 5 0 2}(\mathrm{N})$ & A:K353 (O) & $\mathrm{E}: \mathbf{G 4 8 8}(\mathrm{N})$ & HB & HB & $\checkmark$ \\
\hline
\end{tabular}

187

189 SARS-CoV and a receptor that are not present in ${ }^{27}$ (Table 1). Although the hydrophobic

190 interaction between Y41 (CE2) and T487 (CG2) was not described in ${ }^{27}$, the hydrogen bond 
191 between Y41 and T487 was presented (Table 1; lines 1 and 2). VTR also detected a permuted

192 interaction between atoms of D38 and Y449 (6m0j) and D38 and Y436 (2ajf) (Table 1; lines 3

193 and 7). Moreover, the interactions between $\mathrm{G} 354(\mathrm{O})$ and $\mathrm{G} 502(\mathrm{~N})$ of $6 \mathrm{~m} 0 \mathrm{j}$ and their

194 equivalents in 2ajf appear to be probably detected due to the cutoff-based strategy (Table 1;

195 line 8). Also, VTR detected that G502 interacted with K353: interaction described in ${ }^{27}$. Among

196 the conserved hydrogen bond contacts, four called our attention as they were described in the

197 literature (Figure 4 A-D).
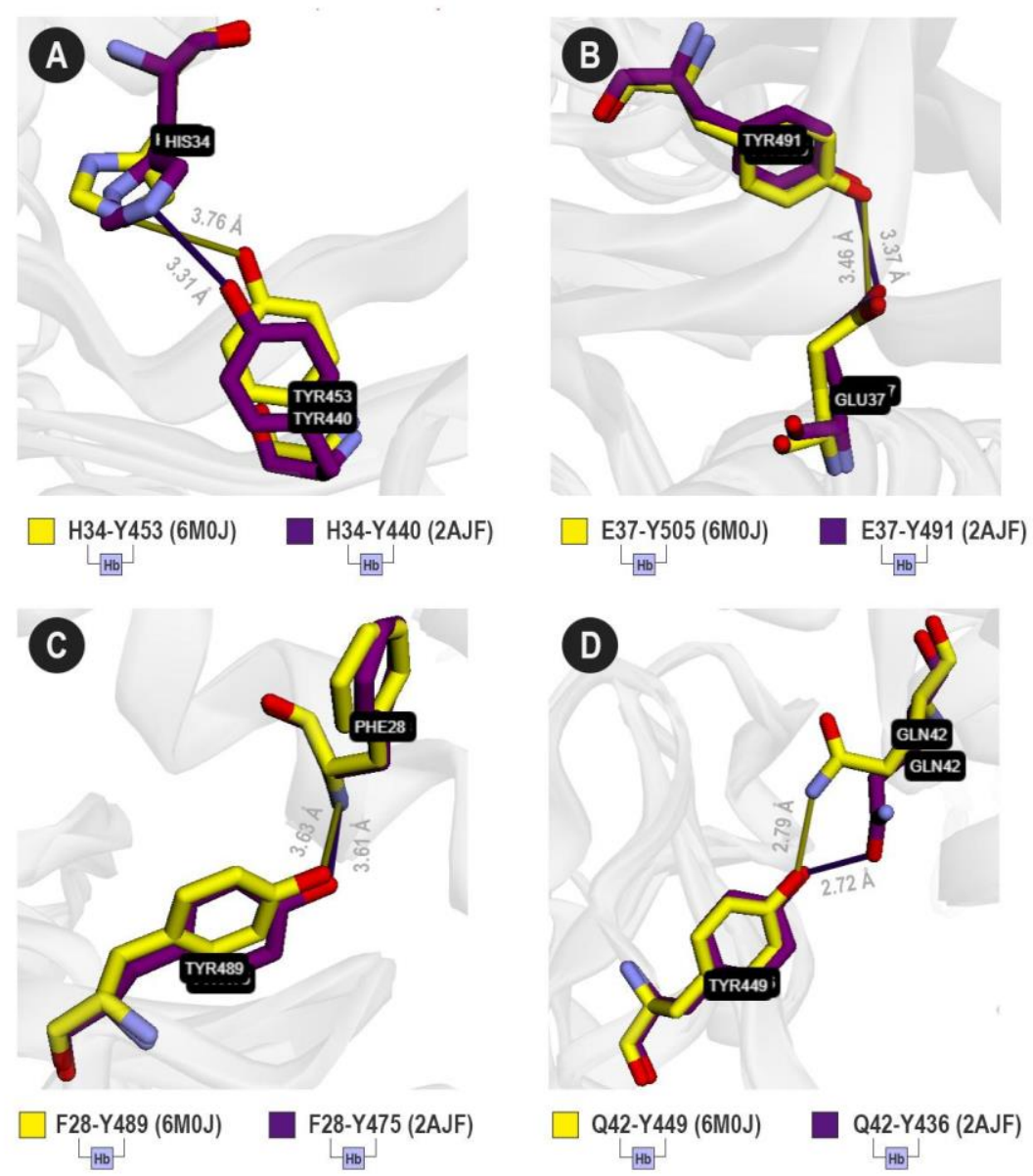

Figure 4. Four analogous contacts between SARS-CoV and ACE2 (purple sticks), and SARS-CoV-2 and ACE2 (yellow sticks). VTR suggests that (a) H34-Y453 (6MOJ) is equivalent to H34-Y440 (2AJF); (b) E37-Y505 (6MOJ) is equivalent to E37-Y491 (2AJF); (c) F28-Y489 (6M0J) is equivalent to F28-Y475 (2AJF); and (d) Q42-Y449 (6M0J) is equivalent to Q42-Y436 (2AJF). Sticks were colored using the yellow/purple color scheme.

For SARS-CoV-2, VTR detected a hydrogen bond between H34 and Y453 that seems to be equivalent to $\mathrm{H} 34$ and Y440 in SARS-CoV (Figure 4A). The same applies to the 
207 following contact pairs: E37-Y505 (6M0J) and E37-Y491 (2AJF) (Figure 4B); F28-Y489

208 (6M0J) and F28-436 (2AJF) (Figure 4C); and Q42-Y449 (6M0J) and Q42-Y436 (2AJF)

209 (Figure 4D). It is important to highlight that besides the interaction between E37 and Y505

210 (Figure 4B), the residue E37 possibly interacts with R403 in 6M0J, but there is no equivalent

211 contact in 2AJF. Also, Lan et al. ${ }^{27}$ described an interaction between $\mathrm{Y} 83(\mathrm{OH})$ and $\mathrm{Y} 489(\mathrm{OH})$

212 of $6 \mathrm{M} 0 \mathrm{~J}$. Besides this interaction, VTR also pointed out that Y489 $(\mathrm{OH})$ interacts with the

213 main-chain atoms of F28. It demonstrates that the cutoff-based strategy used by VTR also has

214 advantages when compared to more restrictive methods. This possible interaction could, in

215 future studies, be better comprehended through molecular dynamics experiments.

216 Case study 3 - Glucose-tolerant $\beta$-glucosidases

$217 \beta$-glucosidases (E.C. 3.2.1.21) are enzymes that act in the last step of the saccharification

218 process, cleaving cellobiose into two molecules of glucose ${ }^{28,29}$. Hence, they are considered

219 very important for the second-generation biofuel industrial applications ${ }^{30-32}$. Besides, the

220 literature has reported that most $\beta$-glucosidases are inhibited in high glucose

221 concentrations ${ }^{33,34}$. Therefore, the design of enzymes more resistant to glucose inhibition has

222 motivated research around the world ${ }^{35}$. Recently, two $\beta$-glucosidases extracted from the

223 marine metagenome of the South China Sea were characterized and evaluated ${ }^{36}$. The first one

224 (Bgl1A) was able to keep its activity even in glucose concentrations up to $1000 \mathrm{mM}^{37}$, being

225 described as glucose-tolerant (a class of $\beta$-glucosidase enzymes with high potential for

226 industrial use). On the other hand, the second one (Bgl1B) was inhibited in concentrations up

227 to $50 \mathrm{mM}^{38}$. Both enzymes have a similar TIM-barrel folding, belonging to the first family of

228 glycoside hydrolases (GH1) ${ }^{39}$. Moreover, they present a sequence similarity higher than

$22950 \%{ }^{40}$. Thus, we decided to submit to VTR the structures of Bgl1A and Bgl1B to evaluate

230 similar contacts and identify possible differences. 
VTR found 375 main contact matches (984 considering hydrophobics), an average

RMSD (for contact matches) of 0.96, and a VTR score of 0.19. From 375 matches, 327 keep

233 the contact type, and 48 change the contact type. The matches that change the contact type are

234 interesting targets for detection of differences in the structures, and potentially can be used to suggest mutations. Thus, such information may guide a biotechnological industry at providing glucose tolerance characteristics to Bg11B through the rational design of enzymes using sitedirected mutagenesis.

After analyzing the results (available in the supplementary material), one contact match called our attention: D41-H57 of Bgl1A (Figure 5A) that corresponds to D41-D57 of Bgl1B (Figure 5B). While Bgl1A presents an attractive contact, Bgl1b presents a repulsive interaction.

241 These contacts are located in the extremities of loop A. Furthermore, this loop has been reported in the literature as necessary for restricting the entrance and exit of the active site in glucose-tolerant $\beta$-glucosidases ${ }^{32}$. Hence, changes in this region could modify the mobility of the protein and could explain the differences in the glucose tolerance of both enzymes.

The literature has reported implications of the topology and dynamics of loop A for the differences in glucose tolerance and inhibition between Bgl1A and Bgl1B ${ }^{32,36}$. To probe the potential of our method for protein engineering, we have checked how considerable were the physical-chemical differences attributed by this point modification at loop A as a whole.

Firstly, we modeled in silico a mutant of Bgl1A (D57H) and a mutant of Bgl1B (H57D).

250 We expected that Bgl1B's mutant present characteristics similar to Bgl1A (i.e., characteristics that could lead to glucose tolerance). As a control, we modeled a Bgl1A's mutant that we expected present characteristics like Bgl1B (i.e., negative characteristics for biofuel production). Then, we inspected the mesoscopic influence of such single amino acid modification at the regional electrostatic surface by Poisson-Boltzmann analysis. 


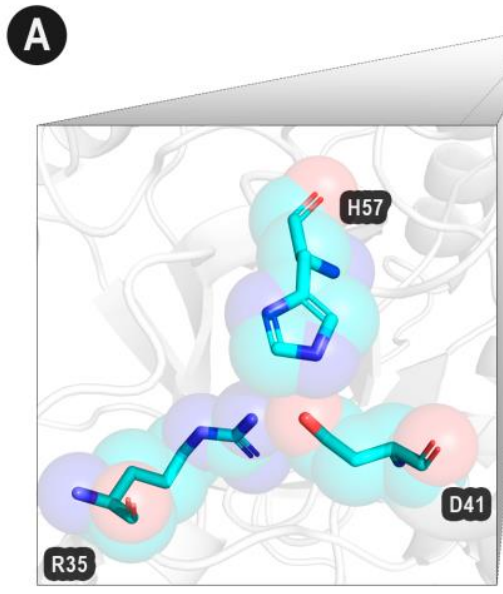

Bgl1 A

C

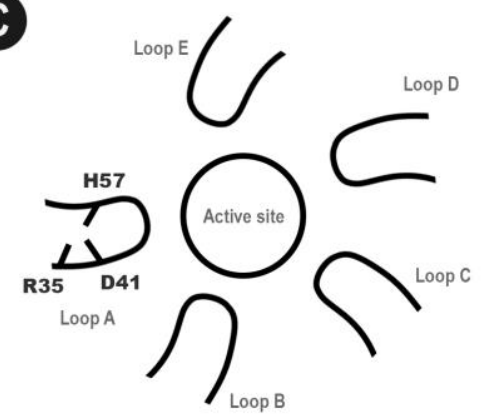

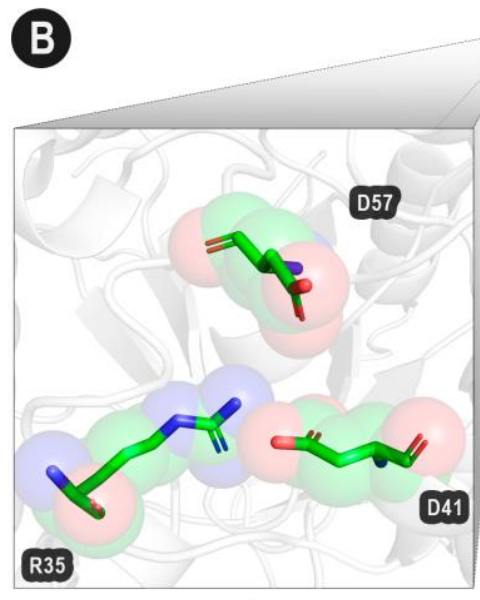

Bgl1B

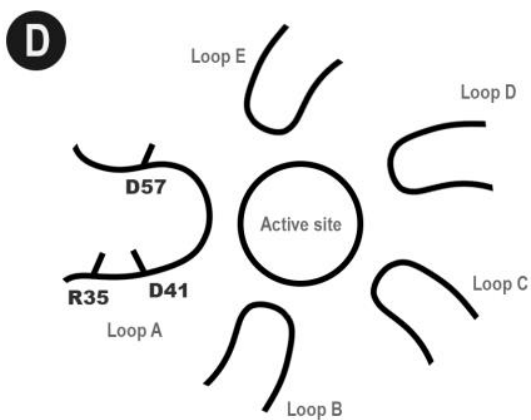

Figure 5. Important contacts in analogous positions between Bgl1 A and Bgl1B. (a) Attractive interaction D41-H57 in loop A of Bgl1A. (b) Repulsive interaction D41-D57 in loop A of Bgl1B. (a-b) Protein backbones are shown as grey cartoons. Amino acid residues are shown as cyan $(\mathrm{Bgl} 1 \mathrm{~A})$ and green (Bgl1B) cartoons. In both proteins, D41 also interacts with R35. (c-d) Illustrative scheme of the importance of these contacts. (c) Bgl1A: H57 performs attractive interactions with D41. (d) Bgl1B: D41 performs repulsive interactions with D57.

\section{PBSA points substantial electrostatic differences}

The estimation of the protonation states at $\mathrm{pH} 7.0$ has recovered the usual HSE protonation for the $\mathrm{H} 57$ in Bgl1A (i.e., a neutral histidine protonated just at the $\varepsilon$ nitrogen atom). This is consonant with the position of the side chain of this histidine in loop A relatively turned to the solvent, with just a marginal contact with the D41 residue at the opposite side. Also, the presence of the positively charged R35 prevents that the H57 local pKa be enough changed by the next D41 to induce a bi-protonation at this histidine (Figure 5). Hence, while the D41-D57 in Bgl1B can be classified as an electrically repulsive contact, the D41-H57 interaction in Bgl1 A is not a charge to charge, but a charge-dipole attractive interaction. Even so, the Poisson- 
274 Boltzmann surface analysis (PBSA) indicates a substantial difference between Bg11A and

275 Bgl1B in electrostatic surface potential in loop A (Figure 6 A-B).
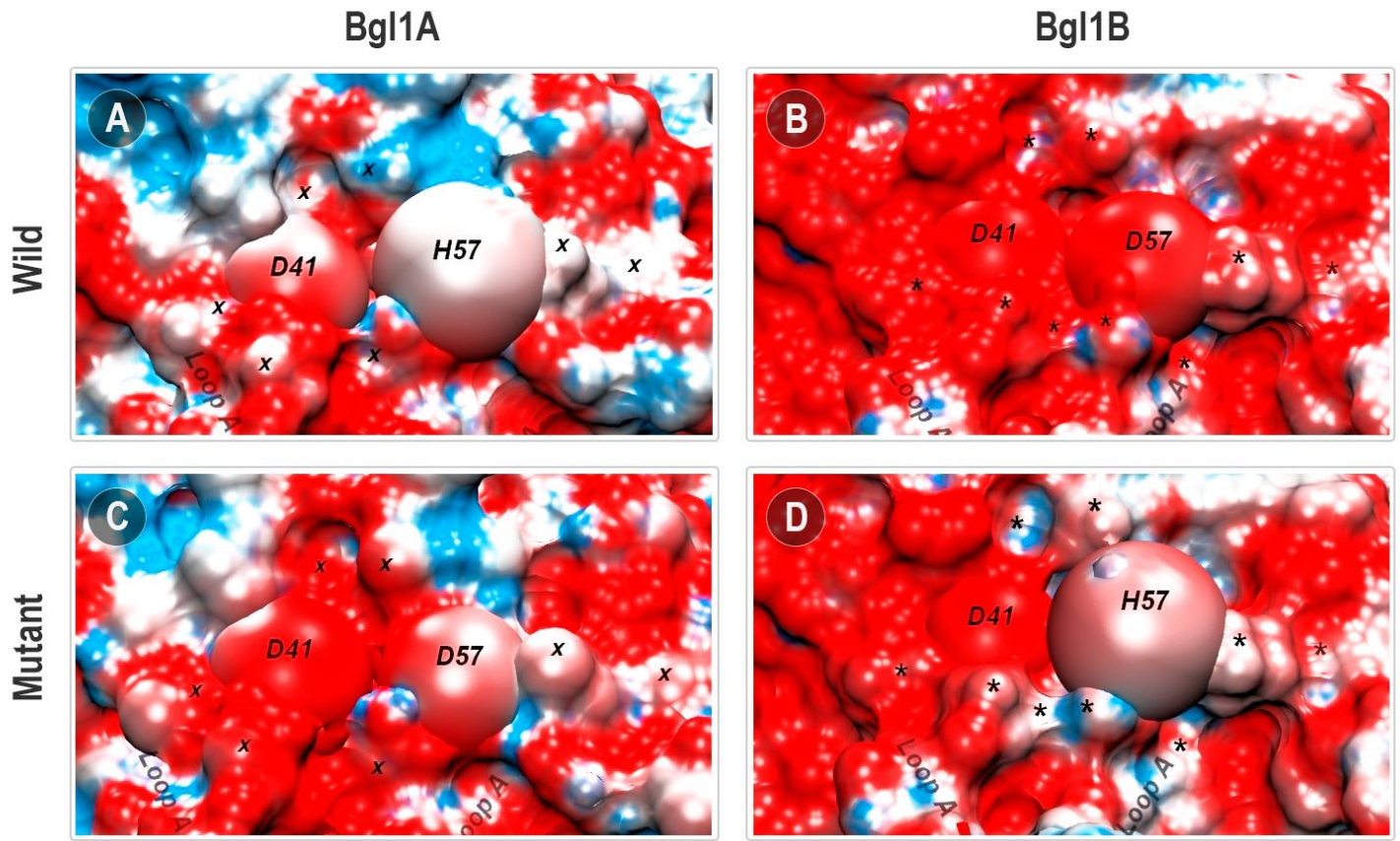

H57D (Bgl1A)

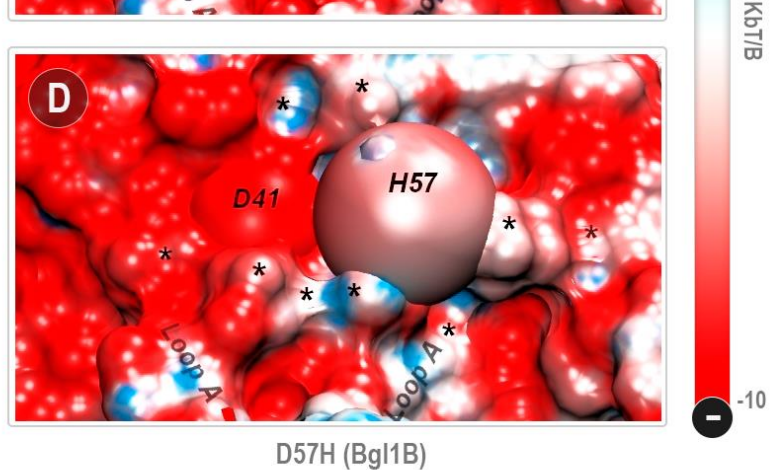

276

Figure 6. Poisson-Boltzmann surface potential at the vicinity between positions 41 and 57 in Bgl1A, Bgl1B, and mutants. (a) Bg1lA; (b) Bgl1B; (c) Bgl1A's H57D mutant; (d) Bgl1B's D57H mutant. The PBSA colors are on a red: white: blue scale for electrostatic potentials around $-10.00:-5.00: 0.00 \mathrm{~kb} / \beta$ unities (being $\mathrm{k}_{\mathrm{b}}$ the Boltzmann constant, $\mathrm{T}$ the $300 \mathrm{~K}$ temperature, and $\beta$ the electron coulomb charge). Residues 41 and 57 are depicted as spheres. Regions whose electrostatic potential is affected by the H57D or D57D permutations are depicted as " $\mathrm{x}$ " for Bgl1A or "*" for Bgl1B.

Indeed, all the topological context around loop A in Bgl1B accumulates a substantial amount of negative electrical potential (red region in Figure 6B). On the other hand, Bgl1A presents a surface with a more neutral electrical potential (blue and white regions in Figure 6A). While the D41-D57 contact appears to be a hot spot of negative charge accumulation in Bgl1B, the correspondent D41-H57 in Bgl1A shares similar neutrality of the surrounding region. The substitutions H57D (Bgl1A) and D57H (Bgl1B) introduce a point inversion of this

290 behavior in both proteins (Figure 6 C-D). Also, these permutations cause electrostatic modifications at specific points of this region. Comparing Figure 6A and Figure 6C (Bg11A 
and its mutant), we can observe an increase of red regions. Contrarily, comparing Figure 6B

293 and Figure 6D (Bgl1B and its mutant), we can observe a reduction of the red regions.

Furthermore, the sites with local potential affected by the permutations appear to be the same in Bgl1A and Bgl1B, spread around loop A. The dynamics and topology of this region are strongly correlated to the functional differences between both proteins ${ }^{32,36}$. In addition, the charge inversions in this region have been correlated to changes in activity and stability ${ }^{41,42}$. All these factors led us to look for a glimpse of the influence of the D41-H57D permutation in the vibrational dynamics of loop A.

\section{Vibrational modulation of loop A}

The representativity of the eigenvectors (Supplementary Figure S1) demonstrates the differences between the PCA recovered fluctuation between loops A of Bgl1A and Bgl1B. Bgl1A fluctuation is more homogeneously represented by the two first eigenvectors (PCs) of loop A, with a fractional eigenvalue of $63 \%$ for the PC1 and $29 \%$ for PC2. On the other hand, the fluctuations at the Bgl1B's loop A are strongly located at PC1, that alone accounts for $82 \%$ of the sum of all eigenvalues. This can indicate that a single kind of movement, with a substantial fugacity from the middle structure at the minima, account for most of the vibratory mobility of Bgl1B's loop A. This is consonant with a local instability caused by the highly repulsive environment at this loop (Figure 6B).

In Figure S1, we can see higher proximity among the lines that represent wild Bgl1B, Bgl1B's mutant, and Bgl1A's mutant. The loop A of the wild Bgl1A presents a more equilibrated and distributed fluctuation between different modes (in concurrence with the more neutral profile in Figure 6 A). In addition, while the H57D exchange is enough to invert the vibrational eigenvalue distribution of the Bgl1A's loop A to the Bgl1B pattern, the same does not occur for Bgl1B with the D57H permutation (Figure S1 A). Hence, the more neutralized electrostatic surface for Bg11A (Figure S1 A) is sensitive to the point insertion of a repulsive 
317 contact at the loop A basis. On the other hand, the strongly negatively charged Bgl1B's loop A

318 is less responsive to neutralization at this single point.

319 Furthermore, the analysis of the distribution of the fluctuations allocated at the two first 320 eigenvectors $(\mathrm{PC} 1+2)$ of loop A shows also considerable differences (Figure S1 B-F). Bgl1A

321 presents higher apparent vibrational mobility concentrated at the C-terminal portion of the Nterminal helix of this loop (residues 45 to 50). In contrast, Bgl1B has its high mobility equally spread along the entire medial portion of loop A (residues 45 to 55). The different electrostatic context (Figure 6 A-B) may explain the vibrational distinctions (Figure S1 A-B). Despite this, the impact in contact patterns, caused by changes in position 57, promotes at least qualitatively standardized modifications around half of loop A (region indicated by lines in Figure S1 C-F). The presence of a negatively charged amino acid in position 57 promotes, both in Bgl1A and Bgl1B's environments, an increase in mobility of positions $44,50,51$, and 57 itself. It also promotes a reduction in the mobility of the region between residues 45 to 49 .

For the D41-D57 repulsive contact, the vibrational movement of both acids is unbalanced, with one moving more or faster than the other ("*" in Figure S1 D-E). On the other hand, the movement involving the D41-H57 contact is more balanced (mainly for Bg11A's context), indicating a more equilibrated vibration again.

\section{$334 \quad$ Implications for protein engineering}

335 The intensity of some of these modifications or changes at the rest of loop A seems to be context-dependent. This agrees with the fact that the permutation of the entire loop A between Bgl1A and Bgl1B was able to revert the glucose tolerant/inhibited behavior, but with poor results for local single amino acid substitutions ${ }^{36}$. Despite this, a single substitution detected by VTR was able to promote vibrational changes with the same pattern at almost half of the extension of this functionally crucial loop. 
All these mentioned facts illustrate a potential use for this tool. If properly combined

342 with electrostatic and mobility patterns detection techniques, such as the APBS and molecular

343 mechanics/PCA here employed, VTR can be useful for rational protein engineering. The

344 integrated use of VTR with computational or experimental tools can be promising to find the topologically minimum modifications that need to be carried at proteins to introduce some desirable characteristics found in other homologous.

For GH1 $\beta$-glucosidases, a protein class extensively used in industry and with a strong interest in the second-generation bioethanol production ${ }^{29}$, this approach shows itself encouraging. Overall, minimal topological modifications represent a promising strategy for suggesting mutations, especially in the beta-glucosidase loop regions that surround the active site. The topology and dynamics of these loops can allow or restrict movements involved in glucose entrance and exit (i.e., glucose tolerance) ${ }^{30,32,36,43}$ or also can affect the thermostability 41-43. These are both examples of industrially desirable characteristics for these and other proteins.

\section{Methods}

356 VTR algorithm receives as input two PDB files and processes them in the back-end using in-

357 house scripts. The files are analyzed in three steps: (i) structural superposition; (ii) contacts

358 computation; and (iii) search for contact matches.

\section{Structural superposition}

360 VTR performs structural alignments between protein pairs using the default parameters of the

361 TM-align algorithm (https://zhanglab.ccmb.med.umich.edu/TM-align). TM-align receives as

362 input two PDB files and returns the coordinates of a superimposed structure. TM-align will return the best alignment possible, and VTR will return a warning informing if contact matches could not be found. 


\section{Contacts computation}

367 We compute the Euclidean distance among all-atom coordinates using in-house Python scripts.

368 The tool identifies five types of possible interactions: hydrophobic, attractive/repulsive,

369 hydrogen bonds, aromatic stacking, and disulfide bonds. A pair of residues are in contact if any

370 atom pair meets the cutoff ranges presented in Table 2.

371 Table 2. Cutoff distances for each contact type. Cutoff values obtained from 1,20,21,44,45.

\begin{tabular}{lcc}
\hline \multicolumn{1}{c}{ Contact type } & Atom classes (residue 1-2) & Cutoff (min-max) \\
\hline Hydrophobic & Hydrophobic - Hydrophobic & $2-4.5 \AA$ \\
\hline Attractive/repulsive & $\begin{array}{c}\text { Positively - negatively charged (attractive) } \\
\text { Positively - positively charged (repulsive) } \\
\text { Negatively - negatively charged (repulsive) }\end{array}$ & $2-6 \AA$ \\
\hline Hydrogen bonds & Acceptor - Donor & $\leq 3.9 \AA$ \\
\hline Aromatic stacking & Ring centroid - ring centroid & $2-6 \AA$ \\
\hline Disulfide bonds & CYS - CYS & $1.5-2.8 \AA$ \\
\hline
\end{tabular}

372

Contact must occur between atoms of two different residues. The atoms involved are described in Table S1. For the detection of aromatic stacking, we determined the centroids of the rings (cutoff 2-6̊). For phenylalanine and tyrosine, we calculate the median coordinate between the atoms CG and CZ for determining the ring centroid. For tryptophan, we used the median coordinate between the atoms CD2 and CE2. For histidine, we determined the median coordinate of the atoms CG, ND1, CE1, NE2, and CD2. residues (until four positions) to remove contacts that compose the structures of $\alpha$-helices.

381 However, through the web tool, users can enable the detection of these contacts (increase processing time). 
383

384

385

386

387

388

389

390

391

392

394

395

396

397

400

401

402

403

404

\section{Search for contact matches}

We used in-house scripts to compare the contacts in analogous positions. We proposed the AVD (average vector distance) metric to measure the distance between contacts and detect contacts matches. AVD is calculated by equation (1):

$$
A V D(P, Q)=\min \left(\frac{D(p 1, q 1)+D(p 2, q 2)}{2}, \frac{D(p 1, q 2)+D(p 2, q 1)}{2}\right)
$$

where $P$ represents the contact between atoms $p 1$ and $p 2$ of protein $A ; Q$ represents the contact between the atoms $q 1$ and $q 2$ of protein $B$; and $D$ is a function that returns the Euclidean distance between atomic coordinates. To detect a contact match between $P$ and $Q$, the $A V D(P, Q)$ should be the lowest value. We determine the VTR score based on equation (2):

$$
\operatorname{VTR}(A, B)=\frac{\sum_{i=1}^{n} A V D_{i}}{C n} \frac{\left(d_{A}+d_{B}\right)}{\left(c_{A}+c_{B}\right)}
$$

where $A$ and $B$ are the proteins analyzed; $n$ is the number of matches found; $C$ is the cutoff determined for the AVD; $d_{A}$ and $d_{B}$ are the number of contacts without match in both proteins; $c_{A}$ and $c_{B}$ are the number of contacts found in each protein. The VTR score is a value between 0 and 1 , where the lower the value, the more similar are two proteins in terms of inter-residue contacts.

\section{Web-based tool}

The VTR web tool was developed using CodeIgniter (https://codeigniter.com/), D3 (https://d3js.org), jQuery (https://jquery.com), DataTables (https://datatables.net), and Bootstrap CSS and JavaScript library (https://getbootstrap.com). 3D structure visualizations are presented using 3Dmol.js ${ }^{46}$. 
405

406

407

408

409

410

411

412

413

414

415

416

417

418

419

420

421

422

423

424

425

426

427

428

429

\section{Case studies details}

For case study 1, we collected the PDBs entries of a sperm whale myoglobin (PDB ID: 1a6m) ${ }^{47}$ and the Paramecium caudatum single-chain and truncated hemoglobin (PDB ID: $1 \mathrm{dlw})^{48}$. For the second case study, we selected SARS-CoV-2 (PDB ID: 6m0j) ${ }^{27}$ and SARS-CoV (PDB ID: 2ajf) ${ }^{49}$ structures in the RCSB PDB. Each PDB file contains two chains A and E, where A represents the cell receptor ACE2 and E the receptor-binding domain (RBD) portion of the virus.

For the third case study, we selected the sequences of the glucose-tolerant GH1 $\beta$ glucosidase of a South China Sea metagenome (Bgl1A; UniProt ID: D5KX75) ${ }^{37}$ and the nontolerant GH1 $\beta$-glucosidase of a South China Sea metagenome (Bgl1B; UniProt ID: D0VEC8) ${ }^{38}$ from Glutantbase ${ }^{50}$. We also constructed two mutants, H57D (Bgl1A) and D57H (Bgl1B), to evaluate VTR ability to propose mutations for enzymes based on differences of contacts (Tables S5-6). The 3D structures of wild (Bgl1A and Bgl1B) and mutant (Bgl1A: H57D and Bg11B: D57H) structures were constructed using SWISS-MODEL ${ }^{51,52}$ (Tables S78). To evaluate the impact of mutations, we estimated the Poisson-Boltzmann surface area (PBSA) with the integrated use of the online versions of the $\mathrm{H}++, \mathrm{PDB} 2 \mathrm{PQR}$, and the Adaptive Poisson-Boltzmann Solver (APBS) tools ${ }^{53-57}$. Details were included in the supplementary material (Additional text S1).

\section{Conclusion}

Herein, we presented VTR, a novel approach with a visual web interface that can be used to analyze, compare, and scrutinize analogous contacts in protein pairs. We explored the tool features with three case studies, where we demonstrated the potential of VTR to shed some light on the mechanisms of topology conservation on phylogenetically related but sequentially distant proteins. We also evaluated contact similarities and dissimilarities on 
430 pharmacologically relevant targets. We suggested the use of our tool to the rational design of

431 proteins with biotechnological applications. Concerning the second case study, both the

432 confirmation of contacts already reported in the literature and the finding of four hydrogen

433 bond matches still not described are promising finds for the future rational design of anti-Sars-

434 Cov-2 drugs. In the last case study, we compared a glucose-tolerant beta-glucosidase enzyme

435 with a non-tolerant one. VTR detected several changes in contact types of analogous positions.

436 Called our attention a change in an attractive contact by a repulsive: D41-H57 of Bgl1A that

437 corresponds to D41-D57 of Bgl1B. We explored the importance of this contact using molecular

438 mechanics minimization and vibrational inspection by principal component analysis. Our

439 results demonstrate that the presence of this contact is vital for the stability of Bgl1A. However,

440 Bgl1B's mutant with a similar interaction was not enough to present similar characteristics of

441 the glucose-tolerant one on a substantial extension. Nevertheless, this case study illustrates the

442 potential of the VTR tool for the rational design of industrial enzymes and gives some glimpses

443 about electrostatic and vibrational aspects of $\beta$-glucosidase enzymes. We hope VTR can be 444 used for understanding differences and similarities between homologous proteins with similar

445 3D structure but differences in sequences. VTR is available at http://bioinfo.dcc.ufmg.br/vtr.

\section{Author contributions}

447 VP and DM developed the webtool and performed the first case study. LXSC and LLB 448 performed the second case study. LHFL and PF performed APBS and molecular dynamics 449 analysis for the third case study. DM wrote the manuscript. VP, PF, LHFL, AVF, and RCMM 450 revised the manuscript. Project design and funding acquisition: RCMM. All authors read and 451 approved the manuscript. 


\section{Availability of data and material}

453 The data underlying this article are available in the article and in its online supplementary 454 material.

\section{Supplementary material}

456 Additional text S1. Material and methods (case study 3)

457 Figure S1. Vibrational mobility pattern recovered by minimization and PCA for loop A of 458 Bgl1A, Bgl1B, and mutants.

459 Table S1. Atoms classes for each type of contact.

460 Table S2. Analogous contacts between proteins with PDB entries 1a6m and 1dlw.

461 Table S3. 1A6M Contacts (Chain A).

462 Table S4. 1DLW Contacts (Chain A).

463 Table S5. Models Assessment (BGL1B).

464 Table S6. Models Assessment (BGL1A).

465 Table S7. Models Assessment (D57H_BGL1B).

466 Table S8. Models Assessment (H57D_BGL1A).

467 Competing interests

468 The author(s) declare no competing interests.

$469 \quad$ Funding

470 This study was financed in part by the Coordenação de Aperfeiçoamento de Pessoal de Nível 471 Superior - Brasil (CAPES) - Finance Code 001. Project grant number 51/2013 $472 \quad 23038.004007 / 2014-82$.

473 Acknowledgments 
474 The authors thank the funding agencies: Coordenação de Aperfeiçoamento de Pessoal de Nível 475 Superior (CAPES), Fundação de Amparo à Pesquisa do Estado de Minas Gerais (FAPEMIG), 476 and Conselho Nacional de Desenvolvimento Científico e Tecnológico (CNPq).

477

478 References

479 1. de Melo, R. C. et al. A contact map matching approach to protein structure similarity $480 \quad$ analysis. Genet Mol Res 5, 284-308 (2006).

2. Chothia, C. \& Lesk, A. M. The relation between the divergence of sequence and structure 482 in proteins. EMBO J. 5, 823-826 (1986).

3. Lesk, A. M. \& Chothia, C. How different amino acid sequences determine similar protein 484 structures: the structure and evolutionary dynamics of the globins. J. Mol. Biol. 136, 225270 (1980).

4. Lesk, A. M. \& Chothia, C. Evolution of proteins formed by beta-sheets. II. The core of the immunoglobulin domains. J. Mol. Biol. 160, 325-342 (1982).

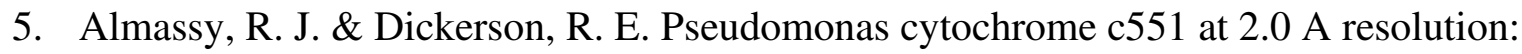
enlargement of the cytochrome c family. Proc. Natl. Acad. Sci. U. S. A. 75, 2674-2678

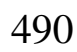

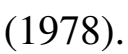

6. Gan, H. H. et al. Analysis of protein sequence/structure similarity relationships. Biophys. $J .83,2781-2791$ (2002).

7. Rost, B. Twilight zone of protein sequence alignments. Protein Eng. 12, 85-94 (1999).

8. Upadhyay, A. Structure of proteins: Evolution with unsolved mysteries. Prog. Biophys. Mol. Biol. 149, 160-172 (2019). (2005). 
498

499

500

501

502

503

504

505

506

507

508

509

510

511

512

513

514

515

516

517

518

519

520

521

522

10. Dill, K. A., Ozkan, S. B., Shell, M. S. \& Weikl, T. R. The Protein Folding Problem. Annu. Rev. Biophys. 37, 289-316 (2008).

11. Dill, K. A. \& MacCallum, J. L. The Protein-Folding Problem, 50 Years On. Science 338, 1042-1046 (2012).

12. Veloso, C. et al. On the characterization of energy networks of proteins. Genet Mol Res 6, 799-820 (2007).

13. Silveira, S. de A., de Melo-Minardi, R. C., da Silveira, C. H., Santoro, M. M. \& Meira Jr, W. ENZYMAP: Exploiting Protein Annotation for Modeling and Predicting EC Number Changes in UniProt/Swiss-Prot. PLoS ONE 9, (2014).

14. Franciscani Jr, G. et al. An annotation process for data visualization techniques. in.

15. Kuntz, I. D. Structure-based strategies for drug design and discovery. Science 257, 10781082 (1992).

16. Barroso, J. R. M. S. et al. Proteus: An algorithm for proposing stabilizing mutation pairs based on interactions observed in known protein 3D structures. BMC Bioinformatics 21, 275 (2020).

17. Melo, R. et al. Finding protein-protein interaction patterns by contact map matching. Embrapa Informática Agropecuária-Artigo Em Periód. Indexado ALICE (2007).

18. Silva, M. F. M. et al. Proteingo: Motivation, user experience, and learning of molecular interactions in biological complexes. Entertain. Comput. 29, 31-42 (2019).

19. Martins, P. M. et al. How to Compute Protein Residue Contacts More Accurately? in Proceedings of the 33rd Annual ACM Symposium on Applied Computing 60-67 (ACM, 2018). doi:10.1145/3167132.3167136.

20. Sobolev, V., Sorokine, A., Prilusky, J., Abola, E. E. \& Edelman, M. Automated analysis of interatomic contacts in proteins. Bioinformatics 15, 327-332 (1999).

21. Neshich, G. et al. STING Millennium: a web-based suite of programs for comprehensive 
and simultaneous analysis of protein structure and sequence. Nucleic Acids Res. 31,

$$
3386-3392 \text { (2003). }
$$

22. Mancini, A. L. et al. STING Contacts: a web-based application for identification and analysis of amino acid contacts within protein structure and across protein interfaces. Bioinformatics 20, 2145-2147 (2004).

23. Pires, D. E. V., de Melo-Minardi, R. C., da Silveira, C. H., Campos, F. F. \& Meira, W. aCSM: noise-free graph-based signatures to large-scale receptor-based ligand prediction.

$$
\text { Bioinforma. Oxf. Engl. 29, 855-861 (2013). }
$$

24. Schrödinger, LLC. The PyMOL Molecular Graphics System, Version 1.8. (2015).

25. Hardison, R. C. Evolution of Hemoglobin and Its Genes. Cold Spring Harb. Perspect. Med. 2, (2012).

26. Zhou, P. et al. A pneumonia outbreak associated with a new coronavirus of probable bat origin. Nature 579, 270-273 (2020).

27. Lan, J. et al. Structure of the SARS-CoV-2 spike receptor-binding domain bound to the ACE2 receptor. Nature 581, 215-220 (2020).

28. Cairns, J. R. K. \& Esen, A. $\beta$-Glucosidases. Cell. Mol. Life Sci. 67, 3389-3405 (2010).

29. Mariano, D. C. B. et al. Characterization of glucose-tolerant $\beta$-glucosidases used in biofuel production under the bioinformatics perspective: a systematic review. Genet. Mol. Res. 16, (2017).

30. Costa, L. S. C. et al. Molecular Dynamics Gives New Insights into the Glucose Tolerance and Inhibition Mechanisms on $\beta$-Glucosidases. Molecules 24, 3215 (2019).

31. Bergmann, J. C. et al. Discovery of two novel $\beta$-glucosidases from an Amazon soil metagenomic library. FEMS Microbiol. Lett. 351, 147-155 (2014).

32. Lima, L. H. F. de et al. Conformational flexibility correlates with glucose tolerance for point mutations in $\beta$-glucosidases - A computational study. J. Biomol. Struct. Dyn. 0, 1- 
20 (2020).

33. Teugjas, H. \& Väljamäe, P. Selecting $\beta$-glucosidases to support cellulases in cellulose saccharification. Biotechnol. Biofuels 6, 1 (2013).

34. de Giuseppe, P. O. et al. Structural basis for glucose tolerance in GH1 $\beta$-glucosidases. Acta Crystallogr. D Biol. Crystallogr. 70, 1631-1639 (2014).

35. Salgado, J. C. S., Meleiro, L. P., Carli, S. \& Ward, R. J. Glucose tolerant and glucose

36. Yang, Y. et al. A mechanism of glucose tolerance and stimulation of GH1 $\beta$ stimulated $\beta$-glucosidases - A review. Bioresour. Technol. 267, 704-713 (2018). glucosidases. Sci. Rep. 5, 17296 (2015).

37. Fang, Z. et al. Cloning and Characterization of a $\beta$-Glucosidase from Marine Microbial Metagenome with Excellent Glucose Tolerance. J. Microbiol. Biotechnol. 20, 1351-1358 (2010).

38. Fang, W. et al. Cloning and characterization of a beta-glucosidase from marine metagenome. Sheng Wu Gong Cheng Xue Bao Chin. J. Biotechnol. 25, 1914-1920

39. Cantarel, B. L. et al. The Carbohydrate-Active EnZymes database (CAZy): an expert

40. Mariano, D. et al. A Computational Method to Propose Mutations in Enzymes Based on modulate the activity and thermal stability of GH1 $\beta$-glucosidases. PloS One 9, e96627e96627 (2014).

42. González-Blasco, G., Sanz-Aparicio, J., González, B., Hermoso, J. A. \& Polaina, J. Directed evolution of $\beta$-glucosidase A from Paenibacillus polymyxa to thermal resistance. J. Biol. Chem. 275, 13708-13712 (2000). 
573

574

575

576

577

578

579

580

581

582

583

584

585

586

587

588

589

590

591

592

593

594

43. Konar, S., Sinha, S. K., Datta, S. \& Ghorai, P. K. Probing the Effect of Glucose on the Activity and Stability of $\beta$-Glucosidase: An All-Atom Molecular Dynamics Simulation Investigation. ACS Omega 4, 11189-11196 (2019).

44. Bickerton, G. R., Higueruelo, A. P. \& Blundell, T. L. Comprehensive, atomic-level characterization of structurally characterized protein-protein interactions: the PICCOLO database. BMC Bioinformatics 12, 313 (2011).

45. Fassio, A. V., Santos, L. H., Silveira, S. A., Ferreira, R. S. \& de Melo-Minardi, R. C. nAPOLI: a graph-based strategy to detect and visualize conserved protein-ligand interactions in large-scale. IEEE/ACM Trans. Comput. Biol. Bioinform. (2019) doi:10.1109/TCBB.2019.2892099.

46. Rego, N. \& Koes, D. 3Dmol.js: molecular visualization with WebGL. Bioinformatics 31, $1322-1324(2015)$.

47. Vojtechovský, J., Chu, K., Berendzen, J., Sweet, R. M. \& Schlichting, I. Crystal structures of myoglobin-ligand complexes at near-atomic resolution. Biophys. J. 77, 2153-2174 (1999).

48. Pesce, A. et al. A novel two-over-two alpha-helical sandwich fold is characteristic of the truncated hemoglobin family. EMBO J. 19, 2424-2434 (2000).

49. Li, F., Li, W., Farzan, M. \& Harrison, S. C. Structure of SARS coronavirus spike receptor-binding domain complexed with receptor. Science 309, 1864-1868 (2005).

50. Mariano, D. et al. Glutant $\beta$ ase: a database for improving the rational design of glucosetolerant $\beta$-glucosidases. BMC Mol. Cell Biol. 21, 50 (2020).

51. Biasini, M. et al. SWISS-MODEL: modelling protein tertiary and quaternary structure using evolutionary information. Nucleic Acids Res. 42, W252-258 (2014).

52. Arnold, K., Bordoli, L., Kopp, J. \& Schwede, T. The SWISS-MODEL workspace: a webbased environment for protein structure homology modelling. Bioinforma. Oxf. Engl. 22, 

195-201 (2006).

599 53. Jurrus, E. et al. Improvements to the APBS biomolecular solvation software suite.

$600 \quad$ Protein Sci. 27, 112-128 (2018).

601 54. Baker, N. A., Sept, D., Joseph, S., Holst, M. J. \& McCammon, J. A. Electrostatics of 602 nanosystems: Application to microtubules and the ribosome. Proc. Natl. Acad. Sci. 98, $603 \quad$ 10037-10041 (2001).

604 55. Dolinsky, T. J., Nielsen, J. E., McCammon, J. A. \& Baker, N. A. PDB2PQR: an 605 automated pipeline for the setup of Poisson-Boltzmann electrostatics calculations. $606 \quad$ Nucleic Acids Res. 32, W665-W667 (2004).

607 56. Anandakrishnan, R., Aguilar, B. \& Onufriev, A. V. H++ 3.0: automating pK prediction 608 and the preparation of biomolecular structures for atomistic molecular modeling and 609 simulations. Nucleic Acids Res. 40, W537-541 (2012).

610 57. Unni, S. et al. Web servers and services for electrostatics calculations with APBS and 611 PDB2PQR. J. Comput. Chem. 32, 1488-1491 (2011). 


\section{Figure legends}

614 Figure 1. VTR workflow. (a) VTR web tool receives as input two PDB files. (b) VTR pipeline analyzes

615 the PDB files in three steps: (i) structural superposition between the PDB files using TM-align; (ii)

616 contacts calculation using cutoff definitions obtained from the literature; and (iii) search for analogous

617 contacts using AVD strategy. (c) VTR returns the contact matches, and users can use them in the web

618 interface. Also, VTR determines the statistics of differences between amino acids in contact.

Figure 2. Contact maps for case studies 1 (a-b), 2 (c-d), and 3 (e-f). Each point represents a contact. Contacts of similar types are shown in the same colors. In the $x$-axes and $y$-axes are shown the residue numbers. (a) PDB ID: 1DLW; (b) PDB ID: 1A6M; (c) PDB ID: 2AJF; (d) PDB ID: 6M0J; (e) Bgl1A; (f) $B g l 1 B$. For $C$ and $D$, we show only contact maps for chain $A$. We consider all contacts for determination of the contact maps, including contacts between atoms of the main-chain of closer residues (such as those present in alpha-helices).

Figure 3. Six analogous contacts between 1a6m (cyan sticks) and 1dlw (green sticks). [Hy] hydrophobic; [Hb] Hydrogen bond; [At] Attractive ionic interaction. (a) V21-V66 (1a6m) and Q13-N43 (1dlw); (b) H36-E38 (1 a6m) and D26-T28 (1dlw); (c) I75-L89 (1dlw) and F48-L64 (1dlw); (d) K102-F106 (1a1m) and A76-T80 (1dlw); (e) L104-S108 (1a6m) and F78-I82 (1dlw); and (f) L89-H93 (1a6m) and L64-H68 (1 dlw). Sticks were colored using the cyan/green color scheme.

Figure 4. Four analogous contacts between SARS-CoV and ACE2 (purple sticks), and SARS-CoV-2 and ACE2 (yellow sticks). VTR suggests that (a) H34-Y453 (6MOJ) is equivalent to H34-Y440 (2AJF); (b) E37-Y505 (6MOJ) is equivalent to E37-Y491 (2AJF); (c) F28-Y489 (6M0J) is equivalent to F28-Y475 (2AJF); and (d) Q42-Y449 (6MOJ) is equivalent to Q42-Y436 (2AJF). Sticks were colored using the yellow/purple color scheme.

638

Figure 5. Important contacts in analogous positions between Bgl1A and Bgl1B. (a) Attractive interaction D41-H57 in loop A of Bgl1A. (b) Repulsive interaction D41-D57 in loop A of Bgl1B. (a-b) Protein backbones are shown as grey cartoons. Amino acid residues are shown as cyan $(B g l 1 A)$ and green (Bgl1B) cartoons. In both proteins, D41 also interacts with R35. (c-d) Illustrative scheme of the importance of these contacts. (c) Bgl1A: H57 performs attractive interactions with D41. (d) Bgl1B: D41 performs repulsive interactions with D57.

645

Figure 6. Poisson-Boltzmann surface potential at the vicinity between positions 41 and 57 in $\mathrm{Bgl} / \mathrm{A}$, Bgl1B, and mutants. (a) Bg1lA; (b) Bgl1B; (c) Bgl1A's H57D mutant; (d) Bgl1B's D57H mutant. The PBSA colors are on a red: white: blue scale for electrostatic potentials around $-10.00:-5.00: 0.00 \mathrm{~kb} / \beta$ unities (being $\mathrm{kb}$ the Boltzmann constant, $\mathrm{T}$ the $300 \mathrm{~K}$ temperature, and $\beta$ the electron coulomb charge). Residues 41 and 57 are depicted as spheres. Regions whose electrostatic potential is affected by the H57D or D57D permutations are depicted as " $\mathrm{x"}$ for Bgl1A or "*" for Bgl1B. 


\section{Figures}

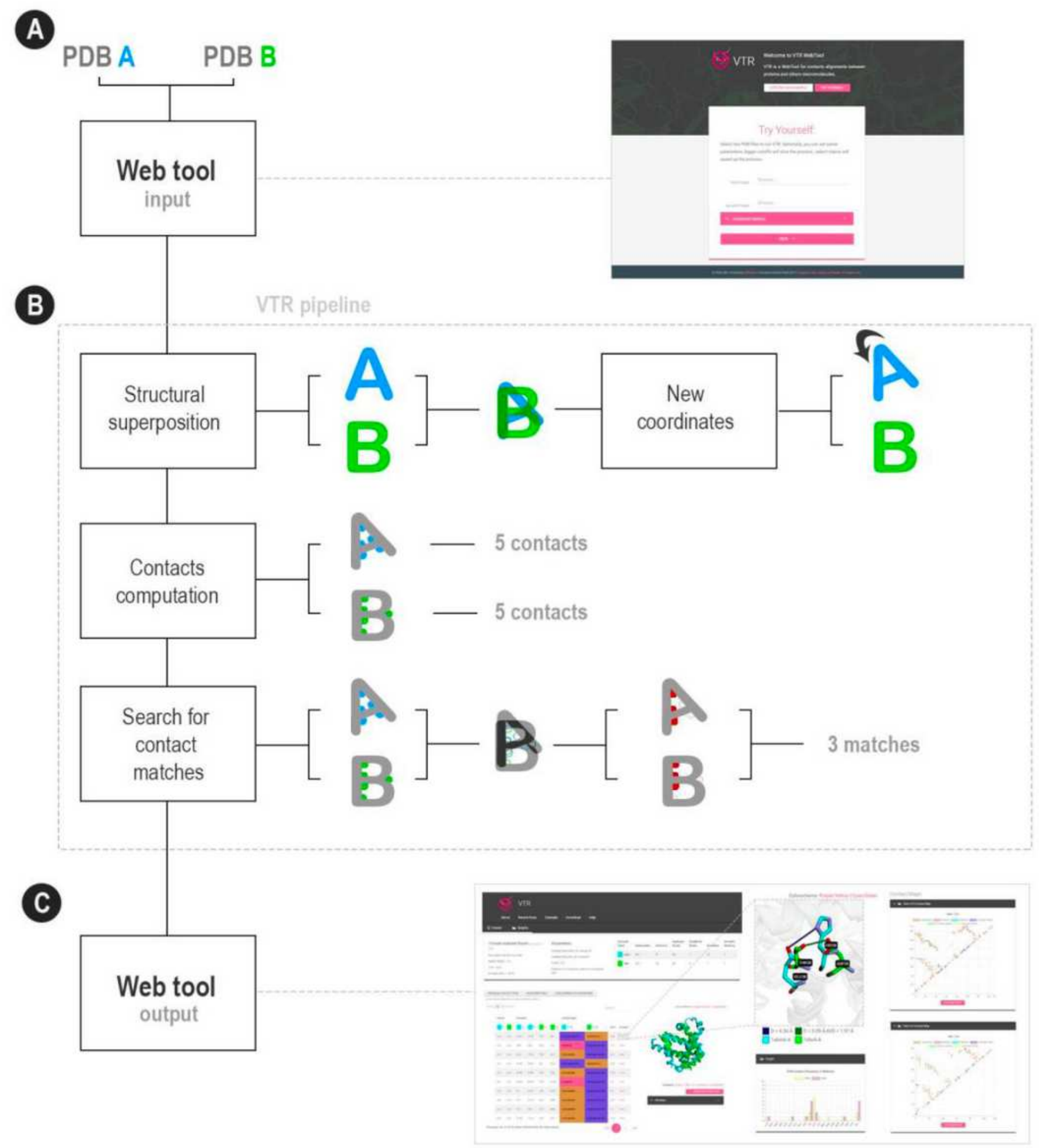

Figure 1

VTR workflow. (a) VTR web tool receives as input two PDB files. (b) VTR pipeline analyzes the PDB files in three steps: (i) structural superposition between the PDB files using TM-align; (ii) contacts calculation using cutoff definitions obtained from the literature; and (iii) search for analogous contacts using AVD 
strategy. (c) VTR returns the contact matches, and users can use them in the web interface. Also, VTR determines the statistics of differences between amino acids in contact.
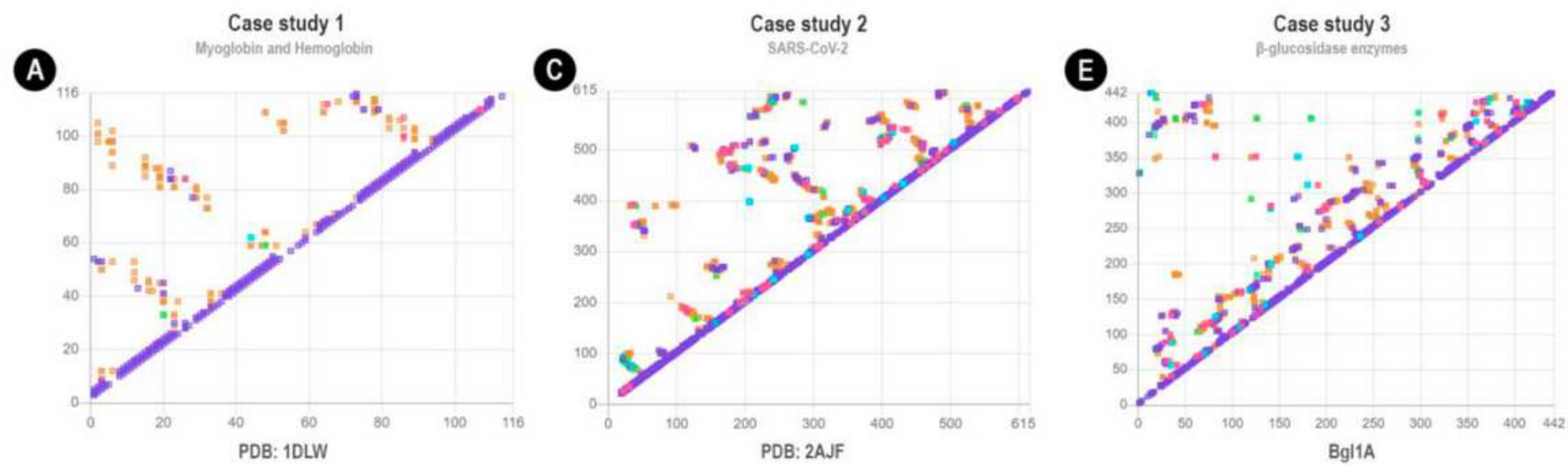

B

(D)
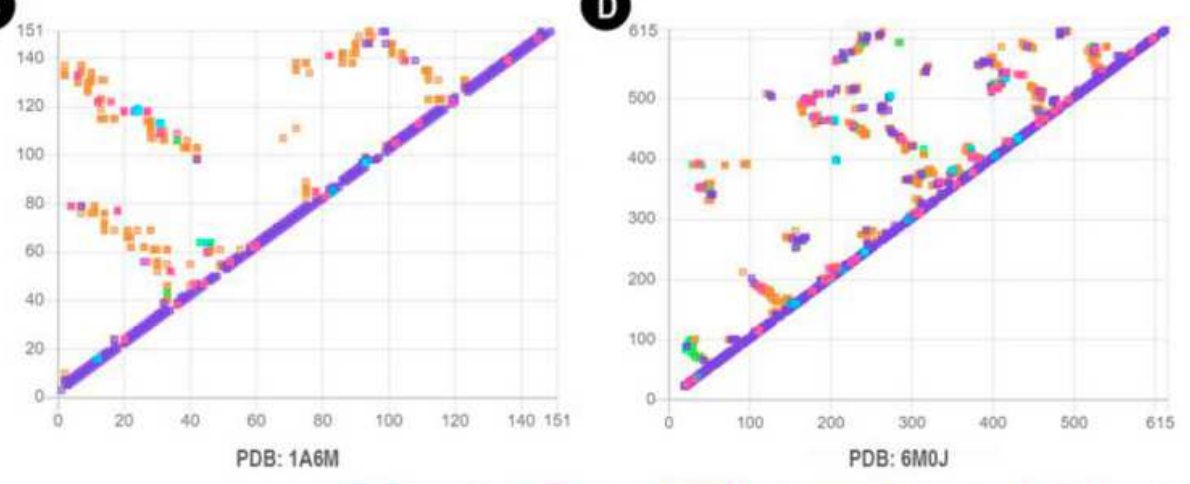

F

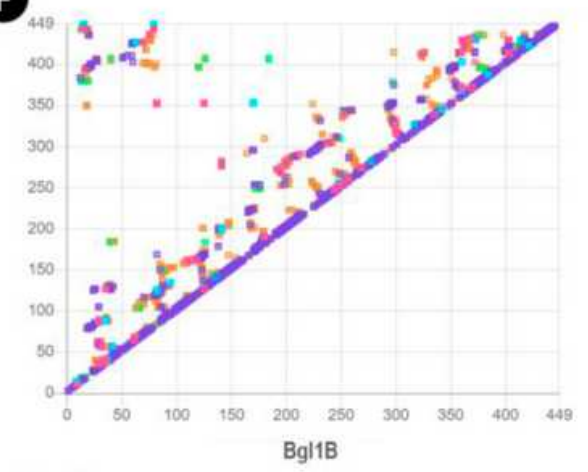

$\square$ Hydrophobic $\square$ Atractive $\square$ Repulsive $\square$ Hydrogen Bonds $\square$ Aromatic Stacking

Disulphide Bonds.

\section{Figure 2}

Contact maps for case studies 1 (a-b), 2 (c-d), and 3 (e-f). Each point represents a contact. Contacts of similar types are shown in the same colors. In the $x$-axes and $y$-axes are shown the residue numbers. (a) PDB ID: 1DLW; (b) PDB ID: 1A6M; (c) PDB ID: 2AJF; (d) PDB ID: 6M0J; (e) Bgl1A; (f) Bgl1B. For C and D, we show only contact maps for chain $A$. We consider all contacts for determination of the contact maps, including contacts between atoms of the main-chain of closer residues (such as those present in alphahelices). 
A

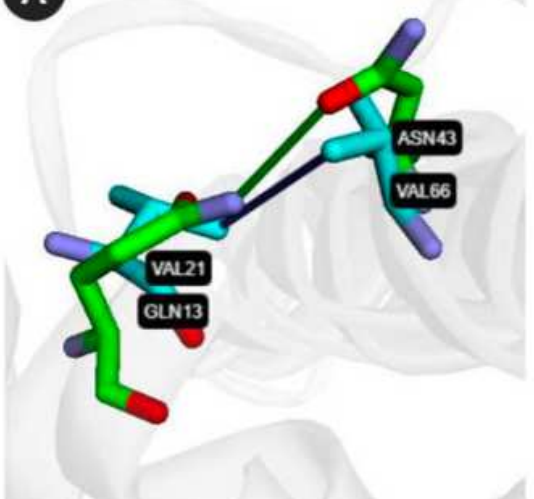

V21-V66 (1A6M)

ᄂ
Q13-N43 (1DLW)

(HO)
B

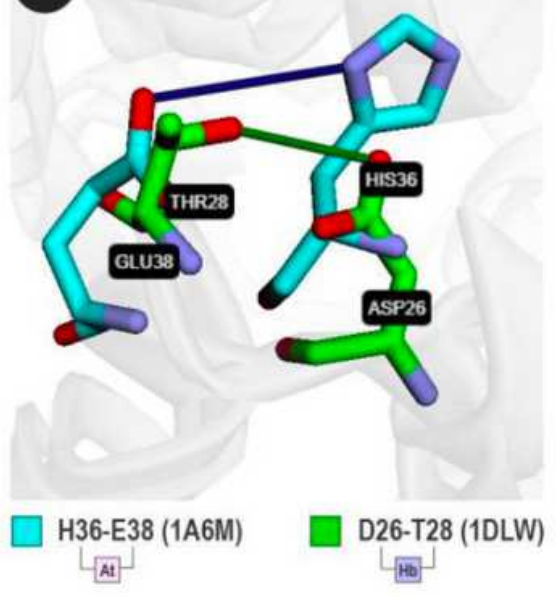

E

D

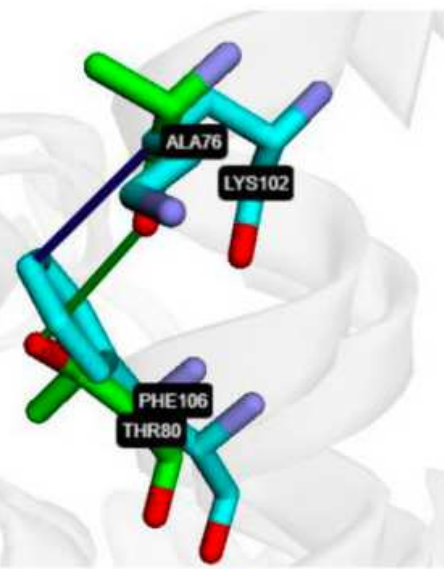

K102-F106 (1A6M)

Hy
A76-T80 (1DLW)

L

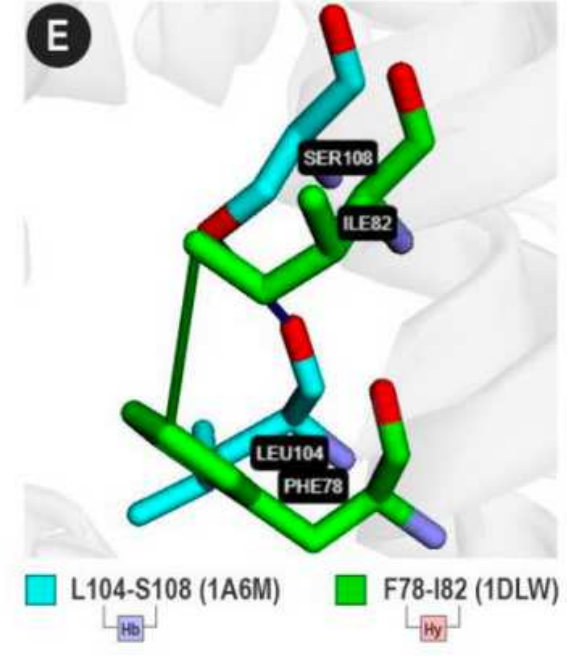

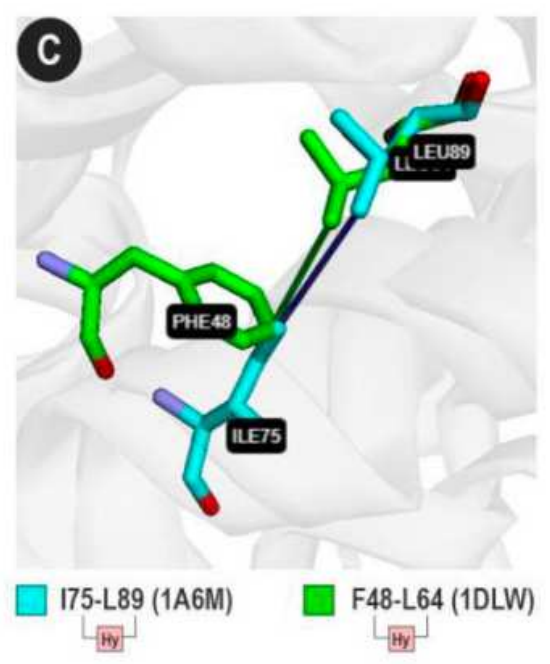

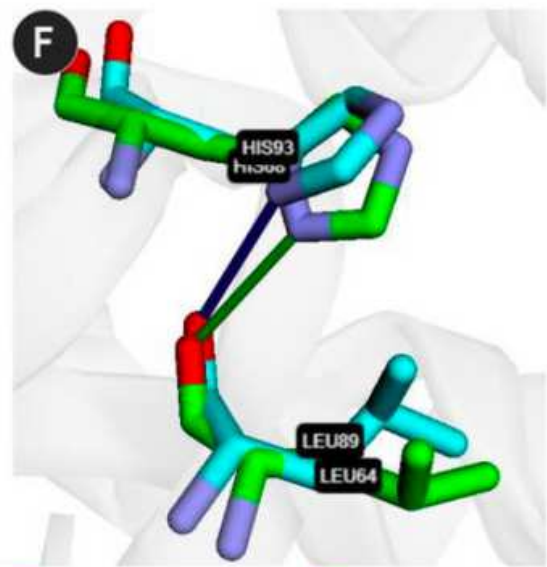

L89-H93 (1A6M)

献

L64-H68 (1DLW)

HB-

\section{Figure 3}

Six analogous contacts between 1a6m (cyan sticks) and 1dlw (green sticks). [Hy] hydrophobic; [Hb] Hydrogen bond; [At] Attractive ionic interaction. (a) V21-V66 (1a6m) and Q13-N43 (1 dlw); (b) H36-E38 (1a6m) and D26-T28 (1dlw); (c) I75-L89 (1dlw) and F48-L64 (1dlw); (d) K102-F106 (1a1m) and A76-T80 (1dlw); (e) L104-S108 (1a6m) and F78-I82 (1dlw); and (f) L89-H93 (1a6m) and L64-H68 (1dlw). Sticks were colored using the cyan/green color scheme. 


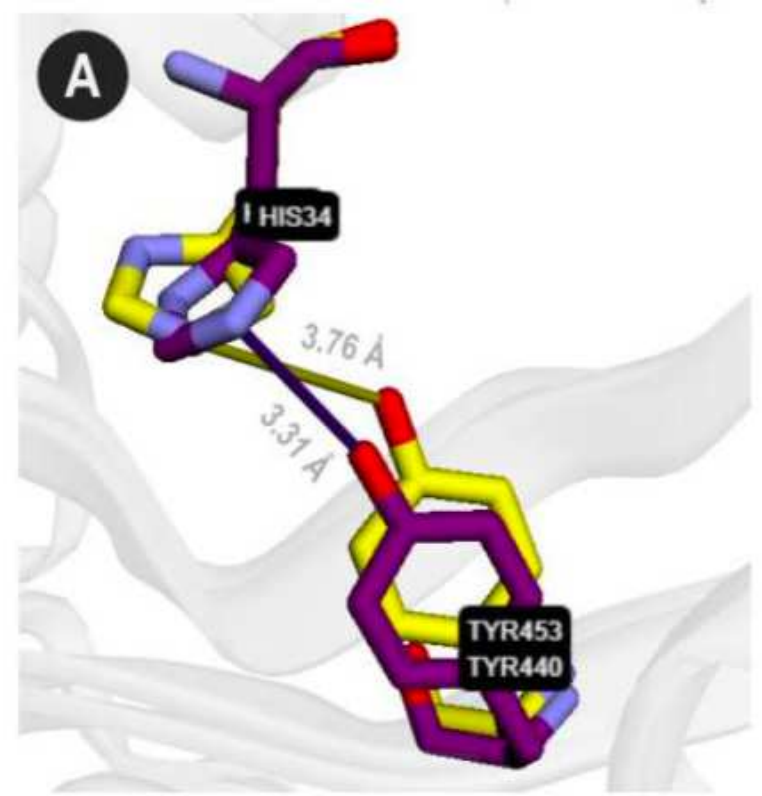

$\square$ H34-Y453 (6M0J) $\square$ H34-Y440 (2AJF) $\left\llcorner_{\mathrm{Hb}} \downarrow\right.$

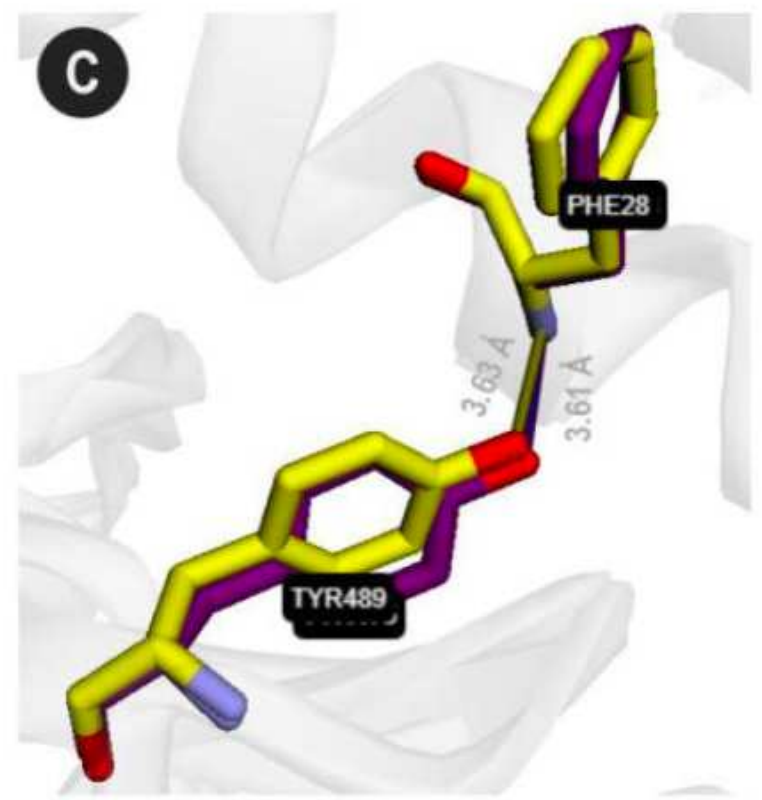

F28-Y489 (6MOJ)

Hb
F28-Y475 (2AJF)

L

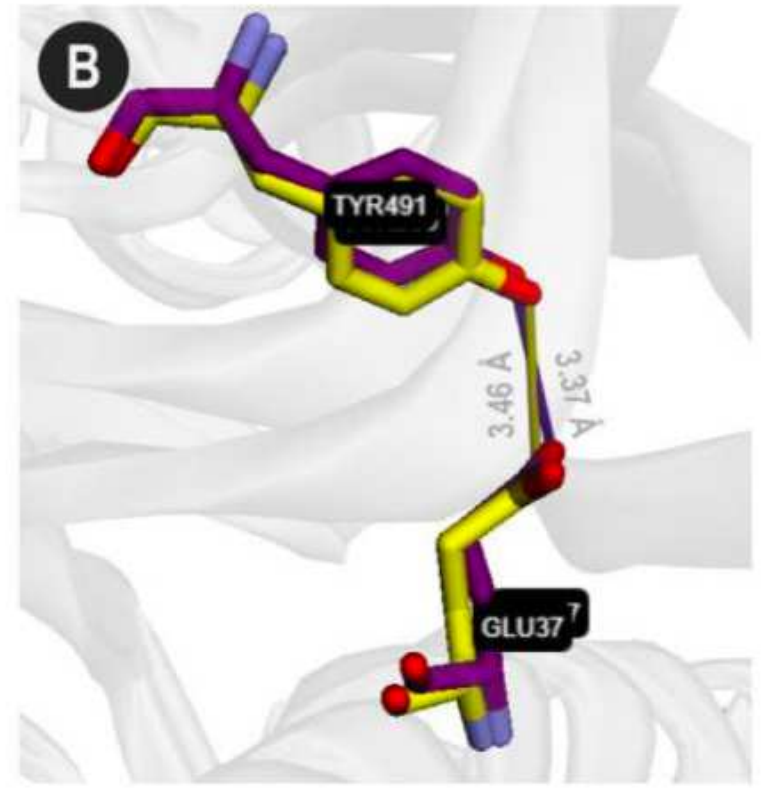

E37-Y505 (6M0J) $\square$ E37-Y491 (2AJF) ᄂ

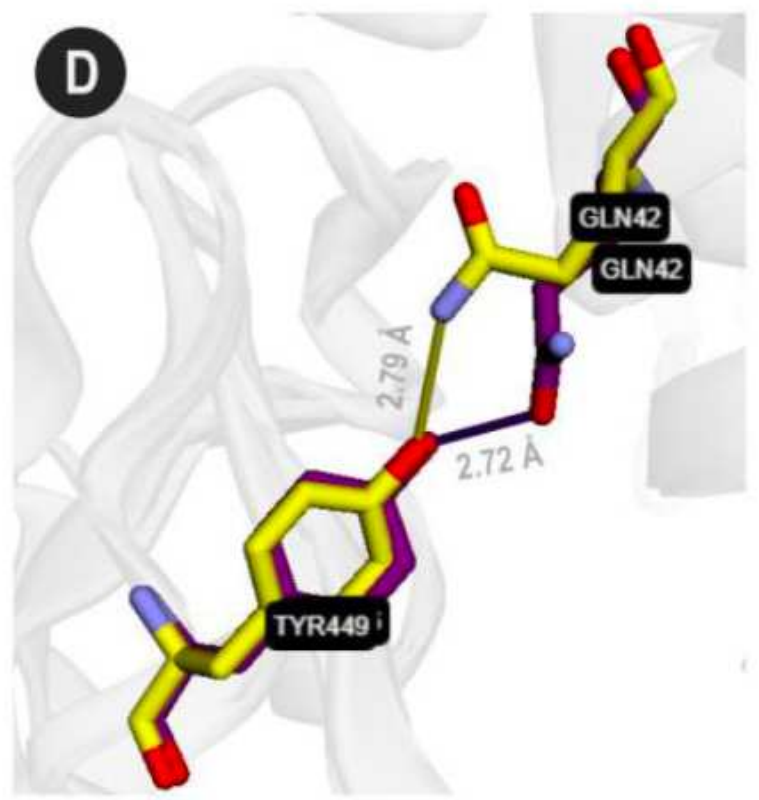

$\square$ Q42-Y449 (6MOJ)

Hb
Q42-Y436 (2AJF)

$-\mathrm{Hb}$

\section{Figure 4}

Four analogous contacts between SARS-CoV and ACE2 (purple sticks), and SARS-CoV-2 and ACE2 (yellow sticks). VTR suggests that (a) H34-Y453 (6MOJ) is equivalent to H34-Y440 (2AJF); (b)E37-Y505 (6M0J) is equivalent to E37-Y491 (2AJF); (c) F28-Y489 (6MOJ) is equivalent to F28-Y475(2AJF); and (d) Q42-Y449 (6MOJ) is equivalent to Q42-Y436 (2AJF). Sticks were colored using the yellow/purple color scheme. 


\section{A}
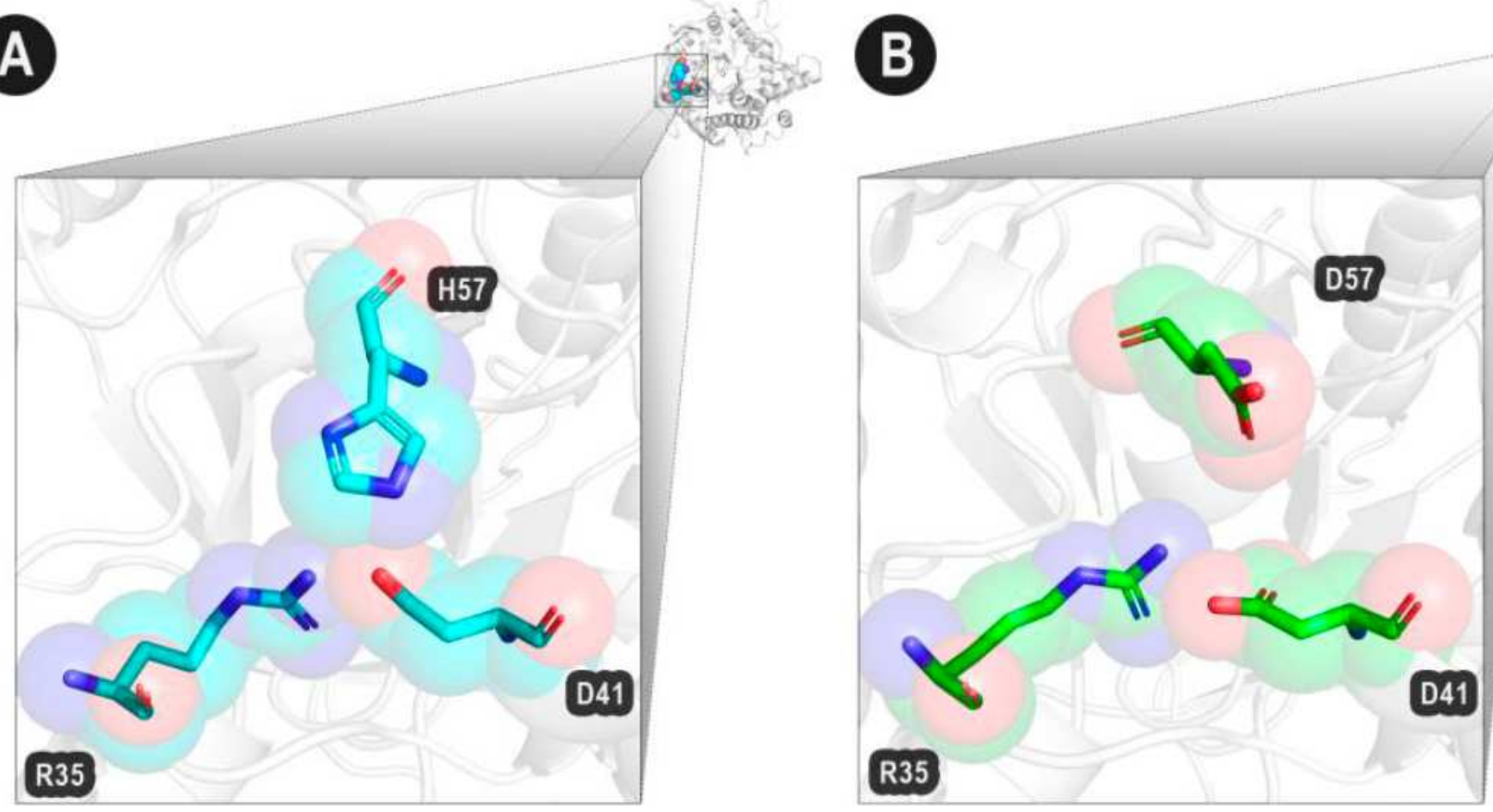

\section{Bgl1 A}

\section{$\mathrm{Bgl1} \mathrm{B}$}
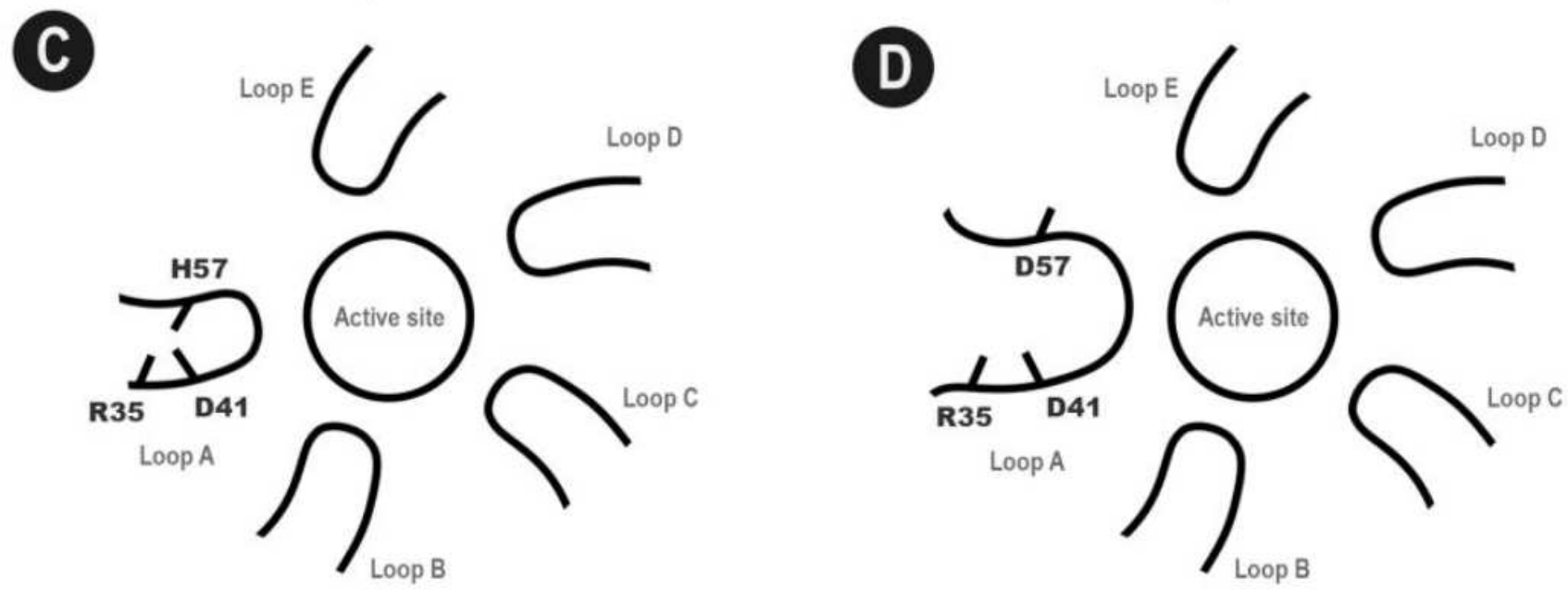

Figure 5

Important contacts in analogous positions between Bgl1A and Bgl1B. (a) Attractive interaction D41-H57 in loop A of Bgl1A. (b) Repulsive interaction D41-D57 in loop A of Bgl1B. (a-b) Protein backbones are shown as grey cartoons. Amino acid residues are shown as cyan (Bgl1 A) and green (Bgl1B) cartoons. In both proteins, D41 also interacts with R35. (c-d) Illustrative scheme of the importance of these contacts. (c) Bgl1A: H57 performs attractive interactions with D41. (d) Bgl1B: D41 performs repulsive interactions with D57. 
Bgl1A
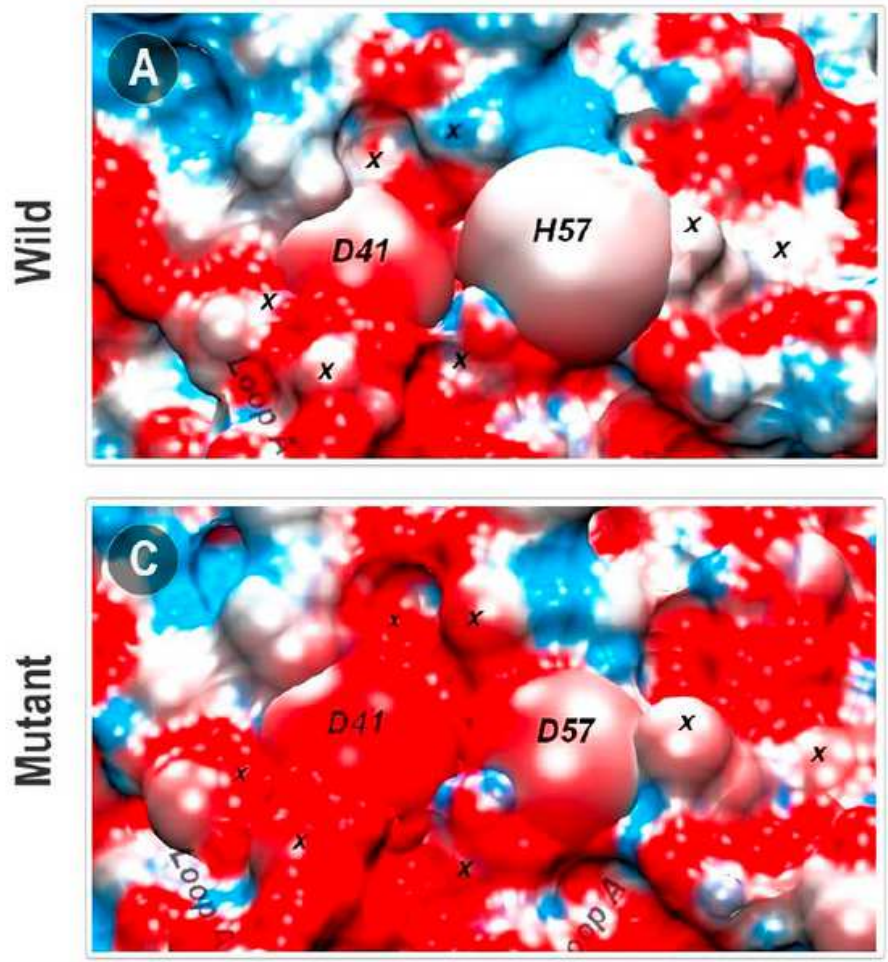

H57D (Bgl1A)
Bgl1B
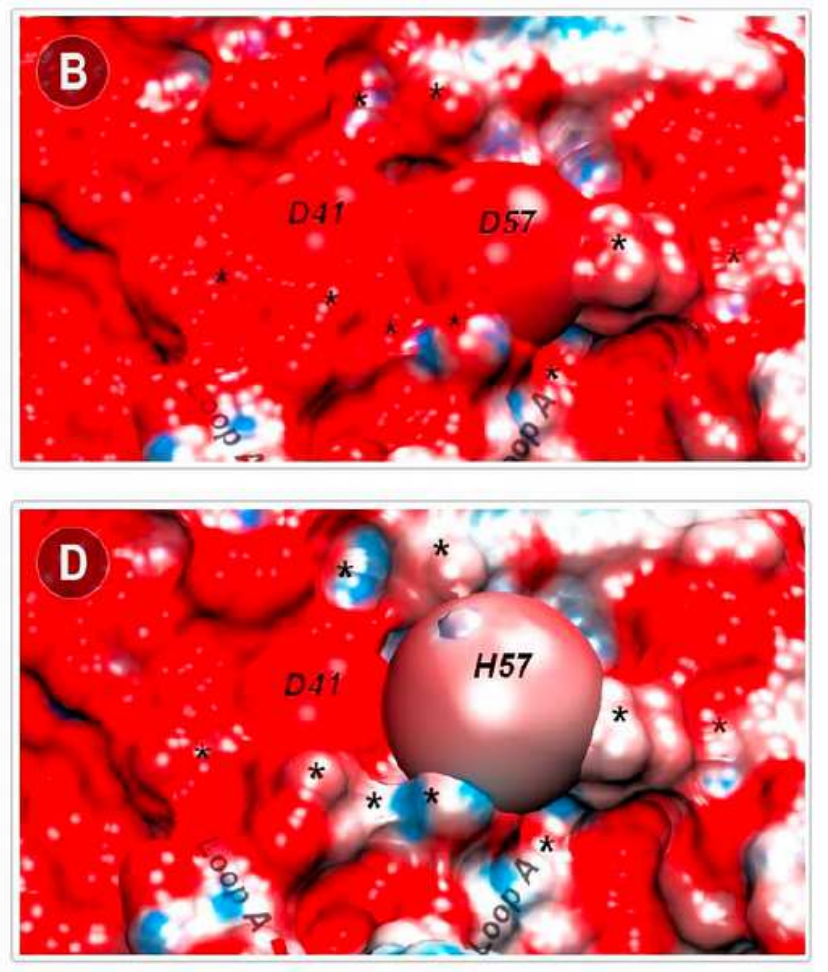

D57H (Bgl1B)

Figure 6

Poisson-Boltzmann surface potential at the vicinity between positions 41 and 57 in Bgl1A, Bgl1B, and mutants. (a) Bg1lA; (b) Bgl1B; (c) Bgl1A's H57D mutant; (d) Bgl1B's D57H mutant. The PBSA colors are on a red: white: blue scale for electrostatic potentials around $-10.00:-5.00: 0.00 \mathrm{kbT} / \mathbb{\nabla}$ unities (being $\mathrm{kb}$ the Boltzmann constant, $\mathrm{T}$ the $300 \mathrm{~K}$ temperature, and $\otimes$ the electron coulomb charge). Residues 41 and 57 are depicted as spheres. Regions whose electrostatic potential is affected by the H57D or D57D permutations are depicted as "x" for Bgl1A or "*" for Bgl1B.

\section{Supplementary Files}

This is a list of supplementary files associated with this preprint. Click to download.

- VTRsupplementarymaterial.pdf 